\title{
DYNAMO-HST Survey: Clumps in Nearby Massive Turbulent Disks and the Effects of Clump Clustering on Kiloparsec Scale Measurements of Clumps
}

\author{
David B. Fisher ${ }^{\star}$, Karl Glazebrook ${ }^{1,2}$, Ivana Damjanov ${ }^{8}$, Roberto G. Abraham ${ }^{7}$, \\ Danail Obreschkow ${ }^{2,4}$, Emily Wisnioski ${ }^{6}$, Robert Bassett ${ }^{1}$, Andy Green ${ }^{9}$, and \\ Peter McGregor ${ }^{10}$ \\ ${ }^{1}$ Centre for Astrophysics and Supercomputing, Swinburne University of Technology, P.O. Box 218, Hawthorn, VIC 3122, Australia \\ ${ }^{2}$ ARC Centre of Excellence for All-sky Astrophysics (CAASTRO) \\ ${ }^{4}$ International Centre for Radio Astronomy Research (ICRAR), M468, University of Western Australia, 35 Stirling Hwy, Crawley, \\ WA 6009, Australia \\ ${ }^{6}$ Max-Planck-Institut für extraterrestrische Physik, Postfach 1312, Giessenbachstr., D-85741 Garching, Germany \\ ${ }^{7}$ Department of Astronomy 83 Astrophysics, University of Toronto, 50 St. George St., Toronto, ON M5S 3H8, Canada \\ ${ }^{8}$ Harvard-Smithsonian Center for Astrophysics, 60 Garden St., Cambridge, MA 02138, USA \\ ${ }^{9}$ Australian Astronomical Observatory, P.O. Box 970, North Ryde, NSW 1670, Australia \\ ${ }^{10}$ Research School of Astronomy and Astrophysics, Australian National University, Cotter Rd, Weston, ACT 2611, Australia
}

Accepted August 2016

\begin{abstract}
We present $\sim 100$ pc resolution Hubble Space Telescope $\mathrm{H} \alpha$ images of 10 galaxies from the DYnamics of Newly-Assembled Massive Objects (DYNAMO) survey of low$z$ turbulent disk galaxies, and use these to undertake the first detailed systematic study of the effects of resolution and clump clustering on observations of clumps in turbulent disks. In the DYNAMO-HST sample we measure clump diameters spanning the range $d_{\text {clump }} \sim 100-800 \mathrm{pc}$, and individual clump star formation rates as high as $\sim 5 \mathrm{M}_{\odot} \mathrm{yr}^{-1}$. DYNAMO clumps have very high SFR surface densities, $\Sigma_{S F R} \sim 1-15 \mathrm{M}_{\odot} \mathrm{yr}^{-1} \mathrm{kpc}^{-2}, \sim$ $100 \times$ higher than in HII regions of nearby spirals. Indeed, SFR surface density provides a simple dividing line between massive star forming clumps and local star forming regions, where massive star forming clumps have $\Sigma_{S F R}>0.5 \mathrm{M}_{\odot} \mathrm{yr}^{-1} \mathrm{kpc}^{-2}$. When degraded to match the observations of galaxies in $z \sim 1-3$ surveys, DYNAMO galaxies are similar in morphology and measured clump properties to clumpy galaxies observed in the high- $z$ Universe. Emission peaks in the simulated high-redshift maps typically correspond to multiple clumps in full resolution images. This clustering of clumps systematically increases the apparent size and SFR of clumps in $1 \mathrm{kpc}$ resolution maps, and decreases the measured SFR surface density of clumps by as much as a factor of $20 \times$. From these results we can infer that clump clustering is likely to strongly effect the measured properties of clumps in high- $z$ galaxies, which commonly have kiloparsec scale resolution.
\end{abstract}

Key words: galaxies: general - galaxies: starburst - galaxies: high-redshift - galaxies: evolution

\section{INTRODUCTION}

Early Hubble Deep Field observations showed that the morphologies of typical galaxies in the distant Universe $(z \gtrsim 1)$ are more irregular than those of local galaxies (Abraham et al. 1996; Conselice et al.

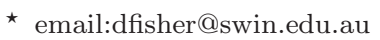

2000; Elmegreen et al. 2005). Subsequent work (e.g. Elmegreen \& Elmegreen 2005; Swinbank et al. 2009; Genzel et al. 2011) has shown that a large fraction of these so-called "clumpy" galaxies are star-forming systems, with extremely high gas fractions (Daddi et al. 2010; Tacconi et al. 2013). The clumps themselves correspond to very young stellar populations with extremely high densities of star formation. Typical clump masses are $\sim 10^{9} \mathrm{M}_{\odot}$ (Elmegreen \& Elmegreen 2005; Swinbank et al. 
2009; Genzel et al. 2011; Wisnioski et al. 2012) with star formation rates up to $\sim 10 \mathrm{M}_{\odot} \mathrm{yr}^{-1}$ per clump. The masses and star formation rates of these clumps are much larger than those of local star-forming complexes.

One clue to the nature of clumpy galaxies is that a large fraction of these systems exhibit a dynamical structure consistent with that of rotating disks whose gaseous content is turbulent (eg. Förster Schreiber et al. 2009; Wuyts et al. 2012; Wisnioski et al. 2011). These two ingredients can lead to large and vigorously star-forming clumps as the product of dynamical instabilities: the high gas dispersions generate large Jeans lengths (e.g. Dekel et al. 2009; Bournaud et al. 2014), while large gas fractions fuel very active star formation.

Our ability to test this model is limited by the fact that observations of star forming regions in high-redshift clumpy galaxies (using either adaptive optics or the Hubble Space Telescope) are typically limited to resolutions of $\sim 1 \mathrm{kpc}$ in the rest frame (e.g. Förster Schreiber et al. 2011a,b; Wuyts et al. 2013), which may be subject to systematic effects of resolution. Simulations with higher resolution predict that clumps viewed at kiloparsec resolution likely are likely composed of several smaller structures, whose sizes are still larger than the Jeans length for the simulated disk (Behrendt et al. 2015). Observationally, finer resolution can be achieved by targetting gravitationally lensed systems (e.g. Jones et al. 2010; Livermore et al. 2012, 2015; Hodge et al. 2012), though uncertainties in lens models limit our ability to interpret these observations. (For example, strong magnification is only achieved in a single direction, namely along the critical curve). Furthermore, it is difficult to construct well-defined samples of lensed galaxies. In general, lensed samples are biased to galaxies with smaller masses and lower star formation rates (e.g. Livermore et al. 2012, 2015).

Clumpy disks were common at redshifts $z \sim 1-3$ (Guo et al. 2015, and references therein). This redshift range corresponds to the epoch at which the co-moving star formation rate density in the Universe was at its peak (Hopkins \& Beacom 2006; Madau \& Dickinson 2014). Therefore, during the main epoch of massive galaxy building, star formation was occurring in a manner that is not well-represented by the modes of star formation seen in nearby galaxies. Not well-represented does not necessarily mean completely absent, however, and there is thus considerable interest in identifying samples of rare local galaxies whose properties are similar to those of high redshift turbulent disks. Such systems can be studied at resolutions higher than the $\sim 1 \mathrm{kpc}$ rest-frame limit that limits most high-redshift observations.

For example, galaxies selected by rest-frame $U V$ brightness and compactness, so-called 'Lyman Break Analogue' galaxies (LBA), have some properties similar to $z>1$ galaxies (Heckman et al. 2005). Recently, Overzier et al. (2009) studied the properties star forming clumps in a sample of LBAs at $z \sim 0.2-0.3$, finding clump sizes of 100 pc. However, based on both spatially resolved kinematics (Basu-Zych et al. 2009) and HST morphologies (Overzier et al. 2008), the vast majority of LBAs resemble the compact dispersion-dominated high- $z$ galaxies found in samples such as that of Law et al. (2009), rather than the turbulent disk systems identified in Förster Schreiber et al.
(2009), Genzel et al. (2011), and Wisnioski et al. (2011). Even if dispersion-dominated systems are disks, they are much more compact that typical massive disks at $z \sim 1-3$ Law et al. (2009). Compact dispersion-dominated systems exhibit star formation that is often driven by ongoing major mergers (Basu-Zych et al. 2009), and it is not obvious that the clumps in LBA galaxies will resemble the clumps in turbulent disks observed at $z \sim 1-3$.

Recently, Green et al. (2010) report the discovery of a sample of galaxies at $z \sim 0.1$ whose properties closely match those of high redshift, turbulent, clumpy disk galaxies. Galaxies in the DYNAMO (DYnamics of Newly-Assembled Massive Objects) sample have high gas fractions of $f_{\text {gas }} \sim$ 20-40\% (Fisher et al. 2014) and $\mathrm{H} \alpha$ velocity dispersions 30$80 \mathrm{~km} \mathrm{~s}^{-1}$ (Green et al. 2010, 2013; Bassett et al. 2014) similar to those of high-redshift turbulent disks. If DYNAMO galaxies do indeed provide local analogs to the high-z systems, then a number of interesting general conclusions about turbulent disks can be drawn from investigations of these local samples. For example, Obreschkow et al. (2015) shows that the specific angular momentum of turbulent disks is lower than in low- $z$ disks of similar mass, suggesting that angular momenta may play an important role in driving dynamical instabilities. Similarly, Bassett et al. (2014) uses DYNAMO galaxies to measure absorption line kinematics in clumpy disks, showing that the kinematical properties of the stars in these galaxies are similar to the kinematical properties of the gas.

In the present paper we report on the properties of massive star forming clumps in 10 DYNAMO galaxies at $z \sim 0.1$ using new Hubble Space Telescope $\mathrm{H} \alpha$ observations. We will compare DYNAMO galaxy morphology to that of high redshift galaxies, in effort to show that resolution degraded images appear similar to high- $z$ galaxies. We then inveistigate the size, luminosity and surface density of clump in DYNAMO maps. We use clump measurements in full, and resolution degraded images to make quantitative tests on the effects of resolution in clumpy galaxies. Throughout this paper, we assume a concordance cosmology with $H_{0}=67 \mathrm{~km} \mathrm{~s}^{-1} \mathrm{Mpc}^{-1}, \Omega_{M}=0.31$, and $\Omega_{\Lambda}=0.69$.

\section{SAMPLE}

\subsection{DYNAMO Survey}

Targets for the present investigation were chosen from the DYNAMO sample which is described in detail in Green et al. (2014).

DYNAMO galaxies were selected from the Sloan Digital Sky Survey (SDSS) DR4 (Adelman-McCarthy \& et al. 2006). Global physical properties for the DYNAMO sample were taken from the JHU-Garching Value Added Catalogues (Brinchmann et al. 2004; Kauffmann et al. 2003; Tremonti et al. 2004), and the sample consists of star forming galaxies that do not show signs of significant active nuclei, based on the BPT diagram. Galaxies were chosen to lie within two redshift windows (centered at $z \sim 0.075$ and $z \sim 0.13$ ) to avoid atmospheric absorption at the wavelength of redshifted $\mathrm{H} \alpha$. 
Table 1. Properties of DYNAMO-HST sample

\begin{tabular}{|c|c|c|c|c|c|c|c|c|c|c|}
\hline Galaxy & $z$ & $\begin{array}{c}\mathrm{SFR} \\
\mathrm{M}_{\odot} \mathrm{yr}^{-1}\end{array}$ & $\begin{array}{c}\mathrm{M}_{\text {star }}^{a} \\
10^{10} M_{\odot}\end{array}$ & $\begin{array}{c}\sigma_{m}(H \alpha)^{a, b} \\
\mathrm{~km} \mathrm{~s}^{-1}\end{array}$ & $\begin{array}{c}V_{2.2}^{a} \\
\mathrm{~km} \mathrm{~s}^{-1}\end{array}$ & $\begin{array}{c}\text { Resolution } \\
\text { pc }\end{array}$ & $\begin{array}{l}\mathrm{A}_{H \alpha}^{a} \\
\mathrm{mag}\end{array}$ & {$[\mathrm{NII}] / \mathrm{H} \alpha$} & $\begin{array}{c}\mathrm{R}_{1 / 2}(\mathrm{H} \alpha) \\
\mathrm{kpc}\end{array}$ & Morphology ${ }^{c}$ \\
\hline G20-2 & 0.1411 & $17.3 \pm 0.7$ & 2.16 & 81 & $166 \pm 10$ & 90 & $0.83 \pm 0.03$ & 0.46 & 2.1 & disk \\
\hline G13-1 & 0.1388 & $26.5 \pm 0.8$ & 1.11 & 76 & $112 \pm 2$ & 93 & $0.50 \pm 0.03$ & 0.19 & 2.6 & merger \\
\hline G14-1 & 0.1323 & $8.3 \pm 0.9$ & 2.23 & 70 & $136 \pm 8$ & 69 & $0.86 \pm 0.04$ & 0.53 & 1.1 & disk \\
\hline G08-5 & 0.1322 & $16.6 \pm 1.0$ & 1.73 & 64 & $243 \pm 15$ & 108 & $1.31 \pm 0.06$ & 0.47 & 1.8 & disk \\
\hline H10-2 & 0.1491 & $25.4 \pm 2.7$ & 0.95 & 59 & $62 \pm 29$ & 117 & $0.21 \pm 0.04$ & 0.20 & 2.6 & merger \\
\hline G04-1 & 0.1298 & $41.6 \pm 2.2$ & 6.47 & 50 & $269 \pm 22$ & 148 & $1.55 \pm 0.05$ & 0.51 & 2.7 & disk \\
\hline D13-5 & 0.0753 & $21.2 \pm 0.9$ & 5.38 & 46 & $192 \pm 2$ & 82 & $1.46 \pm 0.04$ & 0.52 & 2.0 & disk \\
\hline D15-3 & 0.0671 & $13.7 \pm 1.0$ & 5.42 & 45 & $240 \pm 3$ & 51 & $1.77 \pm 0.08$ & 0.35 & 2.2 & disk \\
\hline C13-1 & 0.0788 & $9.9 \pm 0.9$ & 3.58 & 29 & $223 \pm 8$ & 58 & $1.05 \pm 0.10$ & 0.42 & 4.2 & disk \\
\hline A04-3 & 0.0691 & $3.4 \pm 0.7$ & 4.24 & 10 & $218 \pm 2$ & 49 & $1.32 \pm 0.25$ & 0.54 & 3.8 & disk \\
\hline
\end{tabular}

${ }^{a}$ Value taken from Green et al. (2014), methods for determining these properties is described there.

${ }^{b}$ Errors on $\sigma_{m}(H \alpha)$ range $1-3 \mathrm{~km} \mathrm{~s}^{-1}$

${ }^{c}$ Classification of "disks" and "mergers", is done using both kinematics and stellar surface brightness profiles for all DYNAMO-HST galaxies, we discuss this in the Appendix.

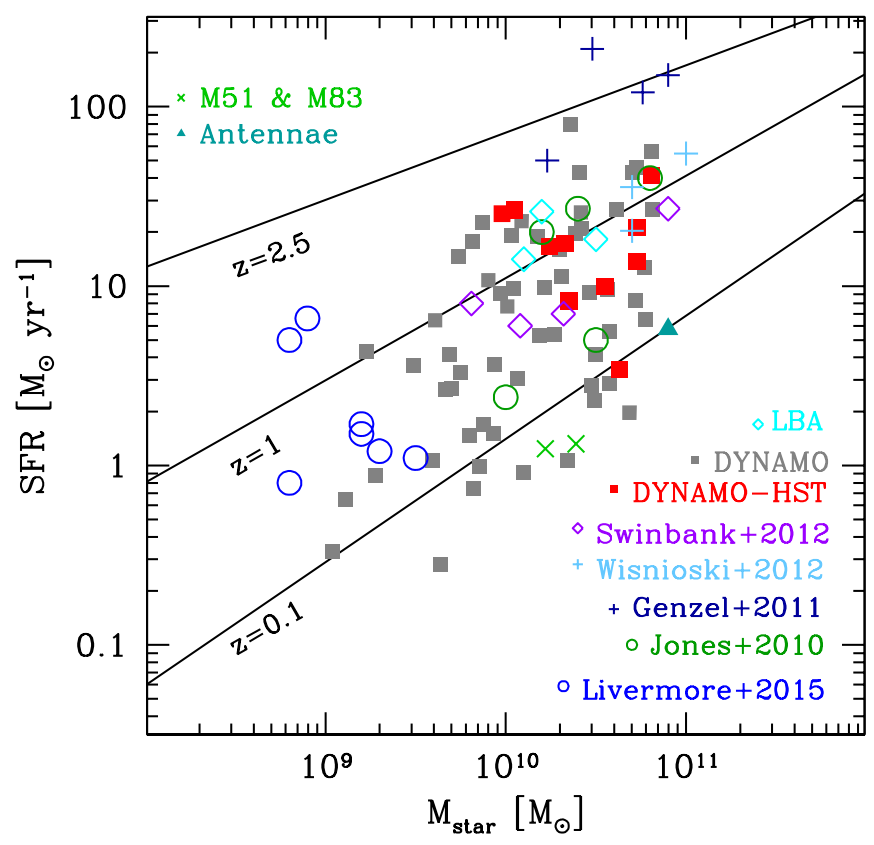

Figure 1. DYNAMO galaxy star formation rates and stellar masses are compared to other samples of clumpy galaxies. Grey squares represent the full DYNAMO survey (Green et al. 2013), and red squares represent the subset studied in this paper. The solid lines represent the main sequence of star formation for $z=$ 0.1,1.0,2.5 (from bottom to top) taken from the analytic fitting function of Whitaker et al. (2012). The ratio of stellar mass-tostar formation in DYNAMO galaxies is very similar to many of those galaxies in which clumps have previously studied, and it is clear that DYNAMO galaxies are very similar to $z \sim 1$ main sequence galaxies. Details of the comparison galaxy samples are described in the Appendix.

\subsubsection{DYNAMO-HST}

In this paper we investigate the properties of a sub-sample of DYNAMO galaxies imaged with the Hubble Space Telescope. This sub-sample (hereafter referred to as DYNAMO$H S T)$ were chosen to be extremely bright in $\mathrm{H} \alpha$ luminosity: $L_{H \alpha}>10^{41.5} \mathrm{erg} \mathrm{s}^{-1}$ in an SDSS fiber, with cor-

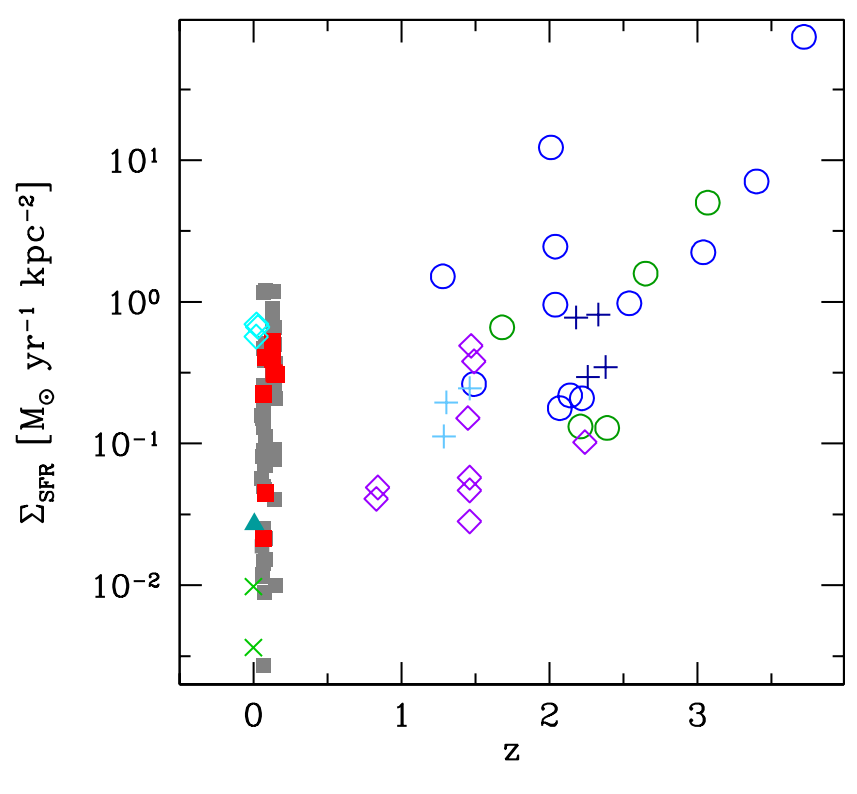

Figure 2. The galaxy star formation rate surface densitty $\left(\Sigma_{S F R}\right)$ is plotted against redshift for the galaxies from Fig. 1. The symbols are the same as in Fig. 1. The star formation rate surface densities of DYNAMO-HST galaxies are similar to that of clumpy galaxies ranging $\mathrm{z} \sim 1.0-2.2$. The star formation rate surface density for local spirals M83 \& M51, as well as the Antennae system are significantly lower than both DYNAMO galaxies and highredshift galaxies.

responding star formation rates of $\sim 10-40 \mathrm{M}_{\odot} \mathrm{yr}^{-1}$. The $\mathrm{H} \alpha$ luminosity cut is chose to match luminosities in of Förster Schreiber et al. (2009); Law et al. (2009); Epinat et al. (2009). All DYNAMO-HST galaxies were initially selected to have rotating, disk-like kinematics in Green et al. (2014), as indicated by significant velocity gradients, and large gas velocity dispersions in the outer disk, $\sigma_{H \alpha} \sim 30-80 \mathrm{~km} \mathrm{~s}^{-1}$ (measured with integral field spectroscopy by Green et al. 2013). Subsequent analysis with higher resolution data and complimentary surface photom- 


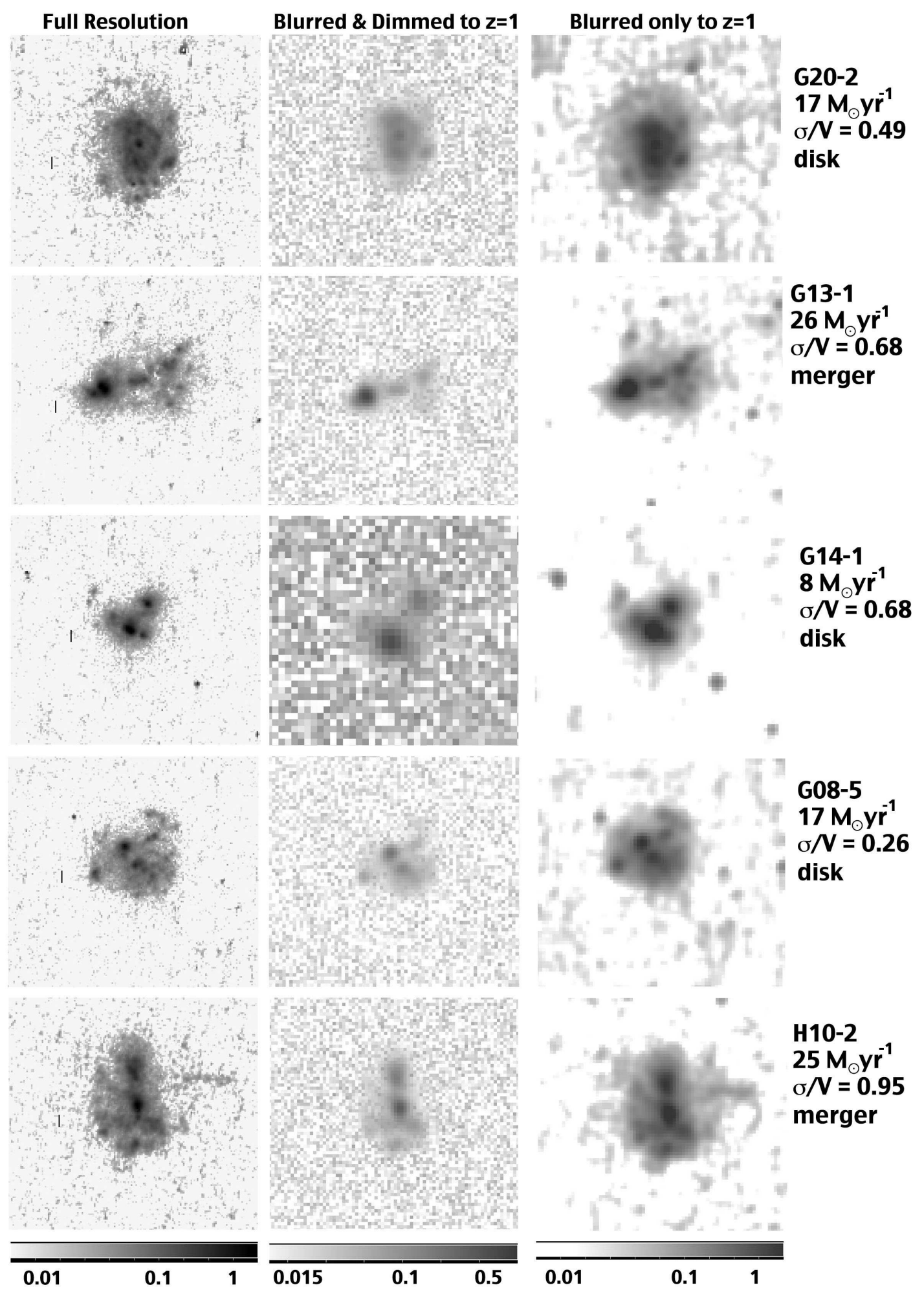

Figure 3. The continuum subtracted $\mathrm{H} \alpha+[\mathrm{NII}]$ maps for the DYNAMO sample galaxies are shown here. Left column shows full resolution maps. The middle column shows maps that have been degraded to simulate $z \sim 1$ observations with blurring, surface brightness dimming and a sensitivity cut similar to high- $z$ AO observations. The right column shows only the effect of blurring maps to match $z \sim 1$ resolution. The FWHM of the blurring corresponds to $1.6 \mathrm{kpc}$ and pixel size $0.8 \mathrm{kpc}$. The maps are arranged according to measured gas velocity dispersion $\left(\sigma_{m}(H \alpha)\right.$ from Green et al. 2013) from highest (top left) to lowest (bottom right). The color bar shows the units of flux in $10^{-18} \mathrm{erg} \mathrm{s}^{-1} \AA^{-1} \mathrm{~cm}^{-1}$. Also the galaxy name, total star formation rate, $\sigma / V$ and optical morphological classification is listed on the far right. A white line is plotted in each panel indicating $1 \mathrm{kpc}$. The DYNAMO sample shows clumpy structures on the scale of a few hundred parsecs. We show the same brightness scale for all maps to emphasize the stark difference in clump prominence between the star forming turbulent disks and the two galaxies with properties more like local spirals (eg. A04-3). Clumps become less prominant with decreasing velocity dispersion and decreasing star formation rate. 


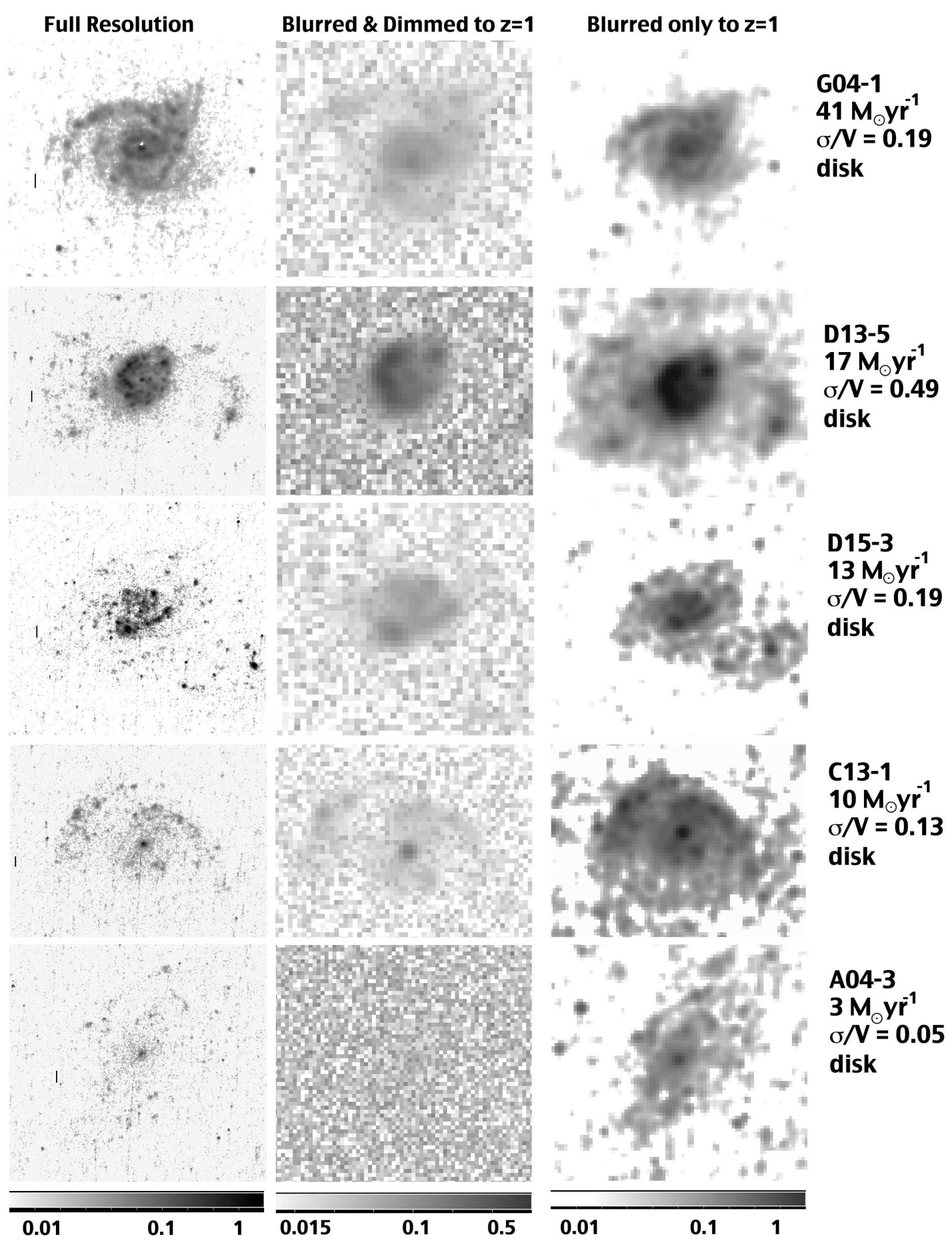

Figure 3. The continuum subtracted $\mathrm{H} \alpha+[\mathrm{NII}]$ maps for the DYNAMO sample galaxies are shown here. Left column shows full resolution maps. The middle column shows maps that have been degraded to simulate $z \sim 1$ observations with blurring, surface brightness dimming and a sensitivity cut similar to high- $z \mathrm{AO}$ observations. The right column shows only the effect of blurring maps to match $z \sim 1$ resolution. The FWHM of the blurring corresponds to $1.6 \mathrm{kpc}$ and pixel size $0.8 \mathrm{kpc}$. The maps are arranged according to measured gas velocity dispersion $\left(\sigma_{m}(H \alpha)\right.$ from Green et al. 2013) from highest (top left) to lowest (bottom right). All images are shown in log scale to emphasize the substructure. The color bar shows the units of flux in $10^{-18} \mathrm{erg} \mathrm{s}^{-1} \AA^{-1} \mathrm{~cm}^{-1}$. Also the galaxy name, total star formation rate, $\sigma / V$ and optical morphological classification is listed on the far right. A white line is plotted in each panel indicating 1 kpc. The DYNAMO sample shows clumpy structures on the scale of a few hundred parsecs. Clumps become less prominant with decreasing velocity dispersion and decreasing star formation rate. 
etry, after the HST program was in complete, found that 2 of the targets H10-2 and G13-1 better resemble mergers (see Appendix for a detailed discussion).

The sample spans $M_{\text {star }}=1-9 \times 10^{10} \mathrm{M}_{\odot}$, and extinction $A(H \alpha) \sim 0.2-1.7 \mathrm{mag}$, both similar to samples of high- $z$ turbulent and clumpy disks (e.g. Förster Schreiber et al. 2009; Swinbank et al. 2012). Overall, galaxies similar to those in DYNAMO-HST are extremely rare in the local Universe, with a space density of $\sim 10^{-7} \mathrm{Mpc}^{-3}$.

In addition to the sample described above, as a control of our method we also targeted one galaxy (DYNAMO A043 ) that has properties that are more typical of low- $z$ galaxies. This object has a star formation rate of $\sim 3 \mathrm{M}_{\odot} \mathrm{yr}^{-1}$ and $\sigma_{H \alpha} \sim 10 \mathrm{~km} \mathrm{~s}^{-1}$.

Thirteen galaxies were imaged as part of the DYNAMO$H S T$ campaign, but observations of three galaxies did not yield usable data. In one case the target was slightly misaligned (spectrally) on the ramp filter, so significant amounts of emission were not mapped. For two galaxies the flux was simply too faint (due to their large distance), and we were therefore unable to identify any substructures with greater than $\sim 1 \sigma$ confidence. The final DYNAMO-HST sample therefore consists of the ten galaxies whose properties are summarized in Table 1.

Figure 1 shows global star formation rate versus stellar mass $\left(M_{\text {star }}\right)$ for the DYNAMO-HST sample, along with the corresponding data for other published samples of clumpy galaxies. In the present paper, star formation rates were calculated from extinction-corrected $\mathrm{H} \alpha$ line luminosity by assuming star formation rate $\left[\mathrm{M}_{\odot} \mathrm{yr}^{-1}\right]=5.53 \times 10^{-42} L_{H \alpha}$ [ $\mathrm{erg} \mathrm{s}^{-1}$ ] (Hao et al. 2011). We calculate the intrinsic $\mathrm{H} \alpha$ extinction using the $\mathrm{H} \alpha$ and $\mathrm{H} \beta$ line ratios from SDSS spectrum. Open circles in Figure 1 correspond to samples of lensed galaxies. The sample of Livermore et al. (2012) is not shown as stellar masses are not available for their galaxies; however the star formation rate of the Livermore et al. (2012) sample are mostly $\sim 0.1-1 \mathrm{M}_{\odot} \mathrm{yr}^{-1}$, with one galaxy $\sim 10 \mathrm{M}_{\odot} \mathrm{yr}^{-1}$. It is evident from Figure 1 that the stellar masses of galaxies in the DYNAMO-HST sample are useful for studies of clump properties of in galaxies with stellar masses in the range $\sim 1-5 \times 10^{10} \mathrm{M}_{\odot}$. Galaxies in this mass range dominated the cosmic star formation rate at $z \sim 1-3$ (Karim et al. 2011).

The average half-light radius of $\mathrm{H} \alpha$ emission in DYNAMO-HST is $R_{1 / 2} \sim 2.5 \mathrm{kpc}$ this is similar to what is found in high redshift galaxies (eg Förster Schreiber et al. 2009). In Figure 2 we compare the star formation rate surface density of DYNAMO-HST galaxies to the same sample of high-redshift galaxies as in Figure 1. For all galaxies we calculate the star formation rate surface density using the total, extintion corrected star formation rate and the $\mathrm{H} \alpha$ half-light radius. DYNAMO galaxies have median $\Sigma_{S F R} \simeq 0.3 \mathrm{M}_{\odot} \mathrm{yr}^{-1} \mathrm{kpc}^{-2}$, for the high- $z$ clumpy galaxy sample we study here the median star formation rate surface density is very similar. Galaxies in Swinbank et al. (2012); Wisnioski et al. (2012); Genzel et al. (2011) all have median star formation rate surface densities of 0.06 $0.5 \mathrm{M}_{\odot} \mathrm{yr}^{-1} \mathrm{kpc}^{-2}$. We note that in Fig. 2 there is a trend that for $z \gtrsim 2.5$ the typical star formation rate surface density increases. Those samples which target higher redshift galaxes, Livermore et al. (2015) and Jones et al. (2010), have median star formation rate surface density of 1.2 and $0.7 \mathrm{M}_{\odot} \mathrm{yr}^{-1} \mathrm{kpc}^{-2}$, respectively. Indeed the results of Figure $1 \& 2$ suggest that the appropriate redshift range for comparison of DYNAMO disks to high- $z$ galaxies is $z \simeq 1-2.5$.

\section{METHODS}

\subsection{New Observations}

$\mathrm{H} \alpha$ flux maps of DYNAMO galaxies were obtained using the Wide Field Camera on the Advanced Camera for Surveys (WFC/ACS) on the Hubble Space Telescope (HST; PID 12977, PI Damjanov). These narrow-band imaging observations were undertaken using the HST ramp filters FR716N and FR782N, which target the $\mathrm{H} \alpha$ emission line with $2 \%$ bandwidth. Central wavelengths in our sample range from 700-754 nm. Data were also taken with the associated continuum filter FR647M. Integration times were $45 \mathrm{~min}$ in the narrow band filter and 15 min with the continuum filter. All images were reduced using the standard HST pipeline.

\subsection{Continuum Subtraction \& $\mathbf{H} \alpha$ Maps}

Our analysis of the clump properties of the DYNAMO$H S T$ sample requires the use of continuum-subtracted maps of $\mathrm{H} \alpha+[\mathrm{NII}]$. In order to subtract the continuum, we first aligned the emission line (FR716N or FR782N) and continuum (FR647M) maps using the positions of point sources. Adjustments were made iteratively until the point sources were aligned within a few percent of a pixel. We then convolved each galaxy's SDSS spectrum with the filter throughput curves for the medium and narrow band filters, and also convolved each SDSS spectrum with two narrow bandpasses just blue and red of the narrowband filter bandpass. This was done in order to estimate the continuum as closely as possible to the $\mathrm{H} \alpha+[\mathrm{NII}]$ emission lines. To avoid contamination by the [SII] doublet, we constructed these bands as follows: First the narrow-band filter throughput was divided into two equal parts so that integrated throughput in each of these is 0.5 . Second, these artificial filters were put then on each side of $\mathrm{H} \alpha+[\mathrm{NII}]$ and convolved with the SDSS spectrum. Finally, the flux from artificial filters is summed to give total narrow-band flux coming from the continuum at the position of $\mathrm{H} \alpha$.

The flux ratio of the medium band filter region to the continuum surrounding $\mathrm{H} \alpha+[\mathrm{NII}]$ was determined, and the flux of the FR647M filter was then scaled by this ratio. We assume a single correction for each galaxy. For most targets the single SDSS 3 arcsec fiber covers the extent of the galaxy. However for larger targets (namely C13-1 and A04-3) we make the assumption that the correction in central 3 arcsec is sufficient to describe the entire galaxies. We remind the reader that our targets are selected to not include AGNs. Finally, the scaled FR647M was subtracted from the narrowband filter maps to construct the final map of $\mathrm{H} \alpha+[\mathrm{NII}]$. The continuum subtracted $\mathrm{H} \alpha+[\mathrm{NII}]$ maps for DYNAMO-HST are shown in Figure 3. Visual inspection of these maps makes it clear that many individual clumps have very bright $\mathrm{H} \alpha$ emission, as do some nuclei. Comparison of the continuum flux of clumps will be the subject of a future paper. 


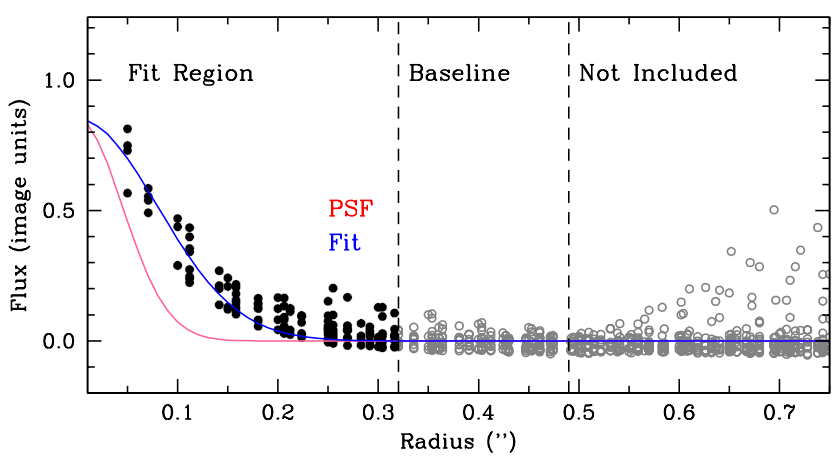

Figure 4. An example light profile of a DYNAMO clumps from galaxy D13-5 is shown. The grey points represent those included in the Gaussian fit. Radial regions are labeled according to whether they are included in the fit, or the baseline determination. The blue line represents a 1-D Gaussian fit to the data, and the red line represents the beam of the image determined on point sources surrounding the galaxy.

\subsection{Identification of clumps}

The precise definition of what a massive star forming clump is, and how it should be identified, varies in the literature (eg. Jones et al. 2010; Genzel et al. 2011; Livermore et al. 2012; Guo et al. 2015). In the present paper we adopt an automated clump identification technique whose purpose is to isolate clumps in a manner similar to the way they have been isolated in high- $z \mathrm{H} \alpha$ maps of clump galaxies (eg. Genzel et al. 2011). Our procedure is a variation of the clump identification strategy employed by Guo et al. (2015), with some minor changes that find their origin in the fact that our observational parameters are not identical to theirs (e.g. our resolution is higher, and our data target $\mathrm{H} \alpha$ emission rather than UV emission from young stars Guo et al. 2015)

The fist step in our procedure was to use an unsharp masking technique to identify emission peaks that are brighter by a factor of at least three compared to the emission in smoothed maps of the same galaxy. Each fullresolution image was divided by a blurred image constructed by convolving the original image with a 1 arcsec Gaussian beam $(\sim 10 \times$ the beam width of $H S T)$. A simple cut was then used to identify the locations of all independent peaks that meet our signal enhancement threshold. For identification purposes we did not place any restrictions on the sizes of the clumps, since our goal is to measure these sizes. Our methodology passes the simplest sanity check of having the brightest set of peaks in the $\mathrm{H} \alpha$ emission maps always corresponding to clumps.

In the sample of DYNAMO-HST galaxies we detected $113 \mathrm{H} \alpha$ peaks whose fluxes were greater than $3 \times$ that of the surrounding area. This corresponds to roughly 11 clumps per galaxy, which is slightly higher than the number of clumps per galaxy reported by Livermore et al. (2012), who found $\sim 7$ clumps in each of their lensed galaxies with similar resolution in the direction of maximum magnification to that of our measurements. For comparison we blurred an archival HST/WFC map of $\mathrm{H} \alpha+[\mathrm{NII}]$ emission from the nearby galaxy M51 to match the physical resolution of a
DYNAMO-HST observation. We then carried out the same clump identification procedure and found 67 emission peaks.

\subsection{Measurement of Clump properties}

To estimate the sizes of star forming clumps, we fitted elliptical Gaussian functions to the 2-D brightness distributions surrounding each peak in the $\mathrm{H} \alpha+[\mathrm{NII}]$ map. This method has been use to estimate the sizes of HiI regions for many years (e.g. Kennicutt (1979)). The reader is referred to Wisnioski et al. (2012) for a detailed discussion of how this method can best be used to estimate the sizes of massive star forming clumps, and to a comparison of the sizes estimated using this technique to sizes determined using isophotal radii.

Figure 4 shows an example radial light profile of a clump from DYNAMO galaxy D13-5. Before fitting the Gaussian, we subtracted the surrounding emission, and this baseline flux was determined iteratively. The initial starting point for this procedure was determined by visual estimation, and this background value was then subtracted from the entire galaxy. For each clump measurement, flux from surrounding clumps was masked, and an initial 2-D Gaussian fit was produced. The baseline background was then re-determined using a region $R>4 \times r_{\text {clump }}$, where $r_{\text {clump }}$ is the half-width of the major axis of the fitted ellipse. The fit was then recomputed iteratively until the fit parameters converged.

The radius of each clump was defined to be the geometric mean of the standard deviations of the major and minor axes ( $a \& b$ respectively) of the 2-D Gaussian function, i.e. $d_{\text {core }}=2 \times \sqrt{a \times b}$. For most clumps this diameter is almost identical to the value returned from a 1-D Gaussian fit to the brightness profile of the clump. However, in cases of very non-circular clumps the size definition chosen always contains the same fraction of the flux, independently from the clump ellipticity. The resolution of each map was measured using ten point sources in the map surrounding each galaxy. We chose to measure this empirically to account for any blurring that was induced by the continuum subtraction. The median beam size of our maps is 0.088 arcsec, which is $160 \mathrm{pc}$ at $z=0.1$. The final clump sizes quoted in this paper have had this beam size subtracted in quadrature from the clump sizes determined from the $\mathrm{H} \alpha+[\mathrm{NII}]$ maps.

Clump fluxes were calculated by integrating the light in the $\mathrm{H} \alpha+[\mathrm{NII}]$ map through an elliptical aperture that is $3 \times$ the size of the core diameter of the fitted ellipse. In the event that a nearby clump overlapped with this region, we masked the nearby clump to calculate the clump properties. For the flux calculation of clumps with masked regions we then interpolate the flux in the missing region using the radial profile. This affects the clump flux at the 10-20\% level. Measured fluxes were then adjusted for extinction, [NII] contribution (determined from SDSS spectra), and distance. The redshift, extinction correction and [NII] correction are each given in Table 1. As mentioned previously, star formation rates were calculated from extinction-corrected $\mathrm{H} \alpha$ line luminosity by assuming $\mathrm{SFR}\left[\mathrm{M}_{\odot} \mathrm{yr}^{-1}\right]=5.53 \times 10^{-42} L_{H \alpha}$ [ $\mathrm{erg} \mathrm{s}^{-1}$ ] (Hao et al. 2011).

Finally, because the properties of central clumps in a galaxy are likely affected by different physical mechanisms (eg. gas in flow to center of galaxy potential), for the analyses in the present paper we have omitted clumps that were coin- 
cident with the galaxy centers determined from the starlight (FR647M) maps. Properties of individual clumps in DYNAMO galaxies are given in Table 2 .

\subsection{Degrading DYNAMO maps to simulate $z \sim 1-2$ observations}

To create a straightforward comparison of the sub-galactic properties of star forming regions we convolve the DYNAMO maps with a Gaussian whose width is set to match the physical resolution of $\mathrm{AO}$ enabled IFU observations on $z \sim 2$ galaxies. Observations of rest frame $\mathrm{H} \alpha$ using AO enabled instruments typically obtain $\sim 0.1-0.3$ arcsec resolution (eg. Genzel et al. 2011; Wisnioski et al. 2012). Across the redshift range $z \sim 1-2$ a median $F W H M \sim 0.2$ arcsec corresponds to $\sim 2 \mathrm{kpc}$ (or a Gaussian standard deviation of $\sim 800 \mathrm{pc}$ ). Note that due to cosmological effects the difference in spatial resolution from $z=1.0$ to $z=2.5$ is only $5 \%$. The typical resolution of our DYNAMO-HST $\mathrm{H} \alpha+[\mathrm{NII}]$ maps is much smaller than this. In the subsequent analysis we will use these blurred DYNAMO maps for comparison to high- $z$ AO samples. We will also use these blurred maps to study how degrading resolution affects measured clump properties.

We also simulate the effects due to decreased sensitivity of high redhsift observations. The includes both the $(1+z)^{4}$ surface brightness dimming and the decreased sensitivity of AO observations of high- $z \mathrm{H} \alpha$ observations. We apply sensitivity cut that is chosen to match the median sensitivity of the $\mathrm{H} \alpha$ maps in SINS survey, $5 \times 10^{39} \mathrm{erg} \mathrm{s}^{-1} \mathrm{kpc}^{-2}$. We fit clump properties both with and with-out the surface brightness effects, and we find that clumps in maps with the $(1+z)^{4}$ cosmological surface brightness dimming to $z=1$ and sensitivity cut have measured core diameters that are the same within $10 \%$ of the blurred core diameter. Calvi et al. (2014) find a similar result. Because clumps are such high surface brightness features they are less effected by redshift dimming. The largest impact is in the ability to detect the clump, we will discuss this in detail later.

Throughout the paper, if a clump is detected in the blurred and dimmed maps then we will use the values from that map. If the clump is only detected in the "blurred only" maps and not in the dimmed map, we will use the blurred only value. This is justified since we find that surface brightness effects have on average no effect on clump sizes, and thus by including these we can study blurring effects with better statistics. Clumps detected in "blurred only" and "blurred + dimmed" maps will be denoted with seperate symbols.

Note that clumps are identified independently each in the blurred DYNAMO maps (Fig. 3 right column) and the full resolution maps (Fig. 3 left column). We do not use the same clump identifications for each. This is done so that we can study, later in this paper, the effect of resolution on clump identification.

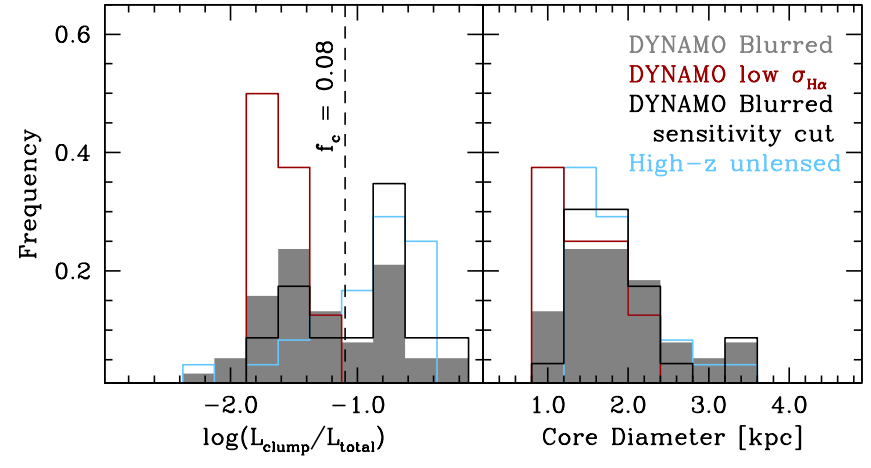

Figure 5. Distribution of the fractional $\mathrm{H} \alpha$ luminosity for each clump to that of the total galaxy, and the distribution of the size of clumps measured in the blurred DYNAMO maps, and the high- $z$ unlensed comparison sample (light blue histogram). The DYNAMO control galaxy (A04-3, red histogram) is counted seperately from the main DYNAMO sample (grey shaded region). The black line represents the DYNAMO clumps in which a sensitivity cut of $5 \times 10^{39} \mathrm{erg} \mathrm{s}^{-1} \mathrm{kpc}^{-2}$ has been applied to mimic that of the SINS survey (Förster Schreiber et al. 2009). The vertical line in the panel showing $L_{\text {clump }} / L_{\text {Total }}$ marks the $8 \%$ line. In both cases the properties of the DYNAMO clumps are well matched to the properties of clumps in high- $z$ galaxies, especially when the sensitivity cut is applied to the DYNAMO sample.

\section{COMPARISON TO HIGH REDSHIFT MAIN-SEQUENCE GALAXIES AND LOCAL ANALOGUES}

\subsection{Comparing DYNAMO galaxies to common definitions of "clumpy" galaxies}

The DYNAMO clumps have $\mathrm{H} \alpha$ luminosities as bright as $\sim 10^{42} \mathrm{erg} \mathrm{s}^{-1}$, corresponding to $\sim 5-10 \mathrm{M}_{\odot} \mathrm{yr}^{-1}$. The median DYNAMO clump has $L_{\text {clump }}(H \alpha) \sim 7.5 \times 10^{40} \mathrm{erg} \mathrm{s}^{-1}$, or $\sim 0.5 \mathrm{M}_{\odot} \mathrm{yr}^{-1}$. To put this in context, the entire $\mathrm{H} \alpha$ luminosity of M $51\left(\sim 2 \times 10^{41} \mathrm{erg} \mathrm{s}^{-1}\right)$, a nearby star-forming disk, may be fainter than a single, bright $\mathrm{H} \alpha$ clump in a DYNAMO galaxy. In the full resolution maps of the DYNAMO$H S T$ galaxies the median clump size for all clumps is $d_{\text {core }}$ 330 pc. Only one clump in the entire sample measures a $d_{\text {core }}<1 \mathrm{kpc}$.

Guo et al. (2015) show, using the CANDELS sample, that defining clumpy galaxies as those in which at least one off-center clump is greater than $8 \%$ of the light is able to distinguish high- $z$ star forming main sequence galaxies from nearby spirals. An important caveat is that this definition is based on UV light from young stars; in this work we have observed DYNAMO galaxies in $\mathrm{H} \alpha$ emission from star forming regions. In the DYNAMO-HST sample 8 out of 10 galaxies satisfy the CANDELS definition of clumpy galaxies. The 2 galaxies that do not satisfy the "clumpy" definition are C 13-1 and A 04-3; these galaxies have the lowest gas velocity dispersions of all galaxies in our sample $\left(\sigma_{H \alpha} \sim 29 \&\right.$ $10 \mathrm{~km} \mathrm{~s}^{-1}$ respectively). They also both have the lowest specific star formation rates in the sample, and therefore are likely to have the lowest gas fractions. Consistent with a basic premise that clumpy star formation is driven by turbulent gas with high gas fractions, those DYNAMO disk galaxies with large gas velocities dispersions $\left(\sigma_{H \alpha}>40 \mathrm{~km} \mathrm{~s}^{-1}\right)$ and 


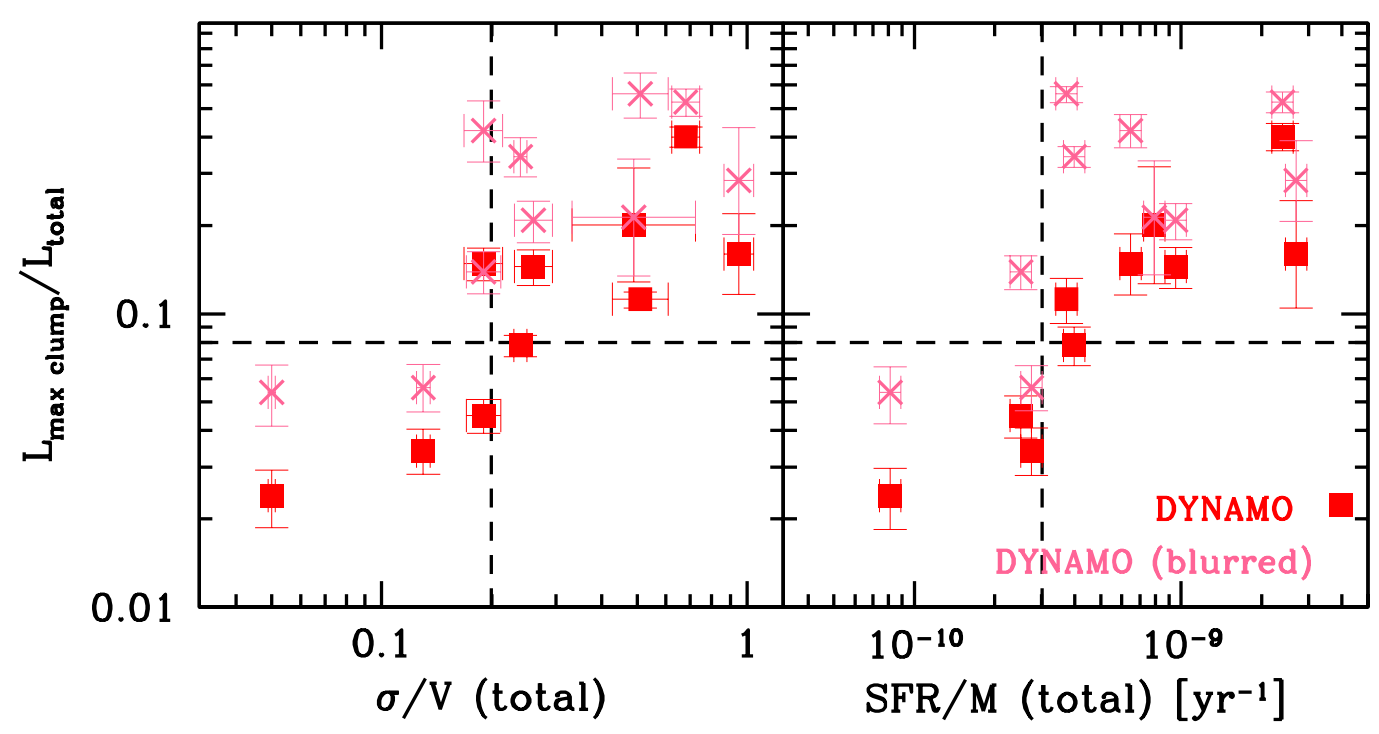

Figure 6. The clump-to-total luminosity ratio of the brightest clump in each galaxy is plotted against both the ratio of $\sigma / V$ and the specific star formation rate for DYNAMO galaxies. The solid red squares represent clumps from the full resolution DYNAMO maps, and the pink crosses represent the same measurement for the DYNAMO maps that have been blurred to match $z \sim 2$ resolution. The horizontal line represents $8 \%$ of the total luminosity. The vertical lines are set to roughly match the point at which the high resolution DYNAMO maps intersect with $8 \%$ line. There is a positive correlation between both the ratio of velocity dispersion to rotation velocity and the specific star formation rate.

high specific star formation rates $\left(\mathrm{SFR} / \mathrm{M}_{*}>3 \times 10^{10} \mathrm{yr}^{-1}\right)$ are found to be "clumpy" galaxies.

In Fig. 3 we compare the properties of clumps in the blurred DYNAMO maps to those published in $z \sim 1-3$ galaxies. In Fig. 5 the 'high- $z$ unlensed' sample refers to the three studies of clump properties in non-lensed galaxies discussed above (Genzel et al. 2011; Wisnioski et al. 2012; Swinbank et al. 2012).

We identify a number of clumps that are comparable in $L_{\text {clump }} / L_{\text {total }}$ to those observed in the high- $z$ Universe. Indeed the distribution of normalized clump fluxes in DYNAMO galaxies, after applying the sensitivity cut, is very similar those observed in high-z galaxies. In Fig. 5 we show that the distribution of sizes of DYNAMO clumps (grey shaded region) in blurred images closely matches that of the high- $z$ unlensed galaxies. We will return to the effects of resolution on clump sizes.

The black lines in Fig. 5 represent the distributions of clump properties that derive from maps in which the sensitivity cut is applied. The average flux ratio $\left(\left\langle L_{\text {clump }} / L_{\text {total }}\right\rangle\right)$ for clumps in the high- $z$ unlensed sample is $16 \%$. The average flux ratio for the sample of DYNAMO-HST clumps that have been blurred and pass the SINS sensitivity cut is almost exactly the same, $17 \%$. A significant minority $(6 / 24)$ of clumps in the high- $z$ unlensed sample have $L_{\text {clump }} / L_{\text {total }}>$ 0.25 , this is similar to the clumps in the blurred DYNAMO$H S T$ sample that have passed the sensitivity cut (4/22). After this sensitivity cut the 2 DYNAMO galaxies, C13-1 and A04-3 do not have observable clumps.

In the right panel of Fig. 5 we show the distribution of clump sizes in the blurred DYNAMO-HST maps and also the clump sizes in high- $z$ unlensed galaxies. Blurred DYNAMO clumps clearly span a similar range and have similar distribution as clumps in high- $z$ galaxies.
In Fig. 6 we show how the clumpiness of DYNAMO galaxies is related to both the ratio of $\mathrm{H} \alpha$ velocity dispersion to rotation velocity $(\sigma / V)$ and the specific star formation rate. Here we trace "clumpiness" with the clump-to-total luminosity ratio of the brightest clump in each galaxy, to preserve the concept in the CANDELS definition of a "clumpy" galaxy. The pink x's mark the values for the blurred DYNAMO clumps, and are thus the most appropriate to compare with high redshift studies. In We find a positive correlation between the clump-to-total flux ratio of the brightest clump and both the specific star formation rate $\left(\mathrm{SFR} / \mathrm{M}_{*}\right)$ and $\sigma / V$. Shibuya et al. (2015) show that the fraction of clumpy galaxies increases with increasing specific star formation rate. Their result is very similar to the right panel of Fig. 6, showing again that DYNAMO galaxies are behaving similarly to $z \sim 1-2$ main-sequence galaxies.

In the following sections when we use the term "clumpy" galaxies from the DYNAMO-HST sample, we will mean those that have clumps that would be detected in the degraded maps, with the median sensitivity level in the SINS survey, and also meet the criteria described in Guo et al. (2015) for the CANDELS survey. To be explicit those DYNAMO galaxies are D13-5, D15-3, G04-1, G08-5, G13-1, G14-1, G20-2, and H10-2. Those galaxies that do not satisfy this definition are A04-3 and C13-1.

\subsection{The size-luminosity correlation of $\mathrm{H} \alpha$ clumps}

For those DYNAMO galaxies that satisfy the definition of "clumpy", the median core diameter of clumps when measured at the full resolution is $\sim 450 \mathrm{pc}$. The majority $(67 \%)$ of clumps have core diameters that range from 200-650 pc in size. Fewer than $10 \%$ of clumps in our sample have clumps 


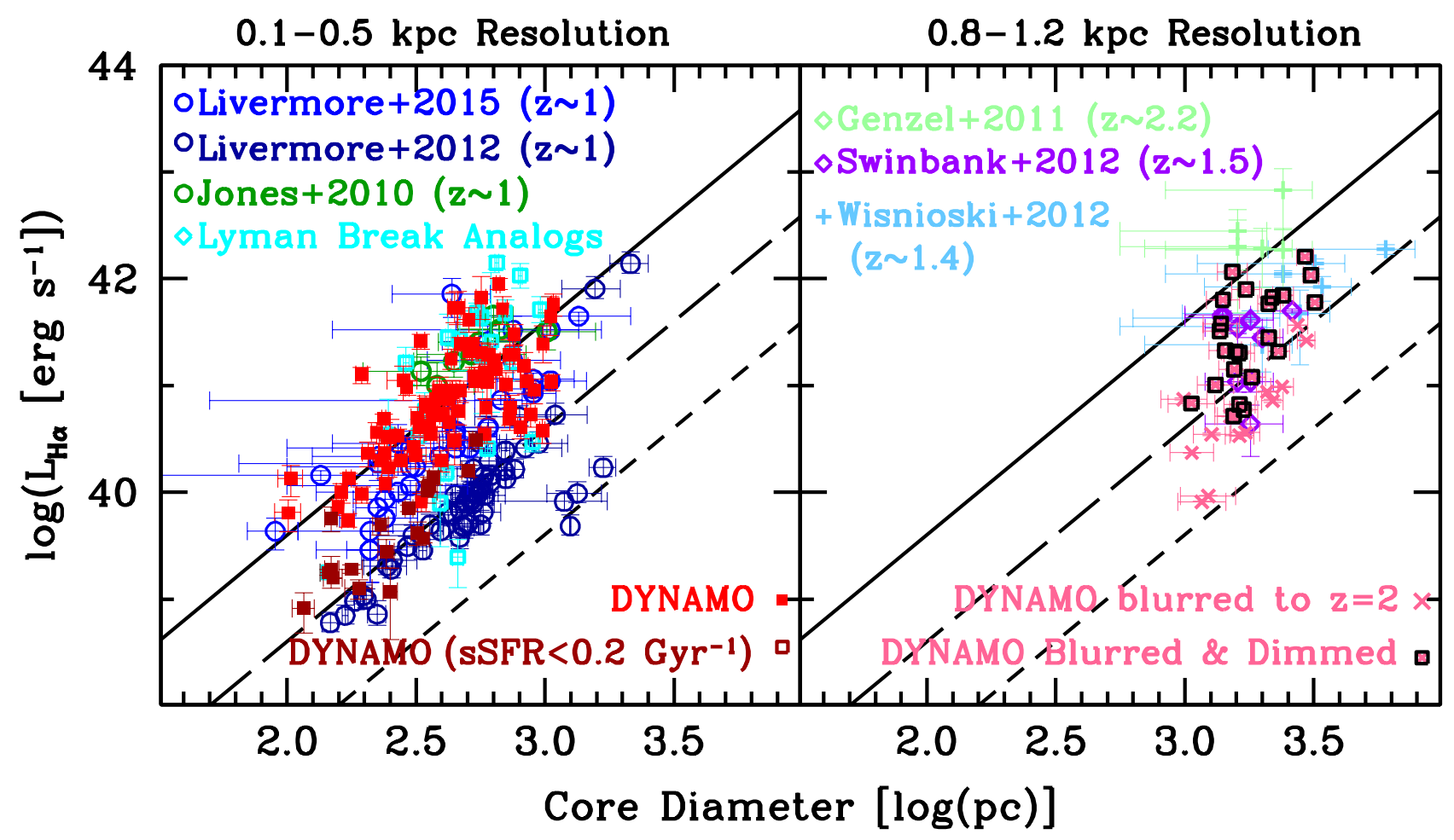

Figure 7. Comparison of position in size-luminosity diagram of DYNAMO clumps to clumps in high redshift galaxies. The left panel shows those samples in which the resolution is of order $\sim 100 \mathrm{pc}$, including lensed galaxies, full resolution DYNAMO-HST galaxies, and a sample of nearby Lyman Break Analogues. The diagonal lines indicate lines of constant surface brightness that are set to match the median DYNAMO clump (solid line) and then a factor of 1/10 (long dashes) and 1/100 (short dashes) lower surface brightness than the DYNAMO median. The right panel shows those samples of high- $z$ galaxies in which the spatial resolution is of order $\sim 1 k p c$. In this panel we also show the clump properties measured on the blurred $\mathrm{H} \alpha$ maps of DYNAMO, with resolution of $\sim 800 p c$, as they would if observed with $H S T$ at $z \sim 2$. Symbol colors and shapes are as described in the key. Clumps in DYNAMO galaxies are as bright as many of the clumps in the unlensed samples of high- $z$ galaxies. DYNAMO clumps are brighter than many of the clumps in lensed galaxies, however are similar sizes.

that are larger than $d_{\text {core }}>800 \mathrm{pc}$, and only 2 out of 79 clumps in clumpy DYNAMO galaxies are larger than $1 \mathrm{kpc}$.

In Fig. 7 we compare the properties of DYNAMO clumps to those in $z \sim 1.0-2.5$ galaxies. The size of DYNAMO clumps, when viewed at full resolution, are very similar to those measured in lensed galaxies, which have similar resolution due to magnification, 100-200 pc. DYNAMO clumps tend to be brighter, than those clumps in the Livermore et al. $(2012,2015)$ samples. Therefore DYNAMO clumps, on average, have higher star formation rate surface density than the clumps typically observed in lensed galaxies.

The star formation rate surface density of clumps in DYNAMO galaxies is mostly contained within $\Sigma_{S F R} \sim 1-$ $10 \mathrm{M}_{\odot} \mathrm{yr}^{-1} \mathrm{kpc}^{-2}$. The median star formation rate surface density of DYNAMO clumps (excluding star forming regions in $\mathrm{C} 13-1$ and $\mathrm{A} 04-3)$ is $\sim 2.3 \mathrm{M}_{\odot} \mathrm{yr}^{-1} \mathrm{kpc}^{-2}$. If we combine the samples of lensed galaxies from Jones et al. (2010), Livermore et al. (2012) and Livermore et al. (2015) the median star formation rate surface density of star forming regions is $\Sigma_{S F R}($ lens $) \sim 0.5 \mathrm{M}_{\odot} \mathrm{yr}^{-1} \mathrm{kpc}^{-2}$, close to an order of magnitude lower than in clumpy DYNAMO galaxies. This is largely due to the samples of Livermore et al. (2012, 2015), which target galaxies with lower total star formation rate. The median star formation rate surface density of clumps in the Jones et al. (2010) sample is $\sim 2 \mathrm{M}_{\odot} \mathrm{yr}^{-1} \mathrm{kpc}^{-2}$, similar to the DYNAMO sample.

In summary, Fig. 7 shows that the clumps in DYNAMO galaxies are consistent in size and luminosity with those of high-redshift galaxies, as well as clumps other galaxies that are considered analogues of high redshift galaxies (LBAs).

\section{SYSTEMATIC EFFECT OF CLUMP CLUSTERING ON BLURRED DYNAMO $\mathrm{H} \alpha$ CLUMPS}

In Fig. 8 we illustrate how the effect of degrading resolution is not simply to blur the properties of a single clump. In this figure arrows indicate a complex of 3 clumps, which when viewed at $z \sim 2$ resolution appears as one clump. At $z \sim 0.1$ resolution, the three clumps have an average core diameter of $600 \mathrm{pc}$, each being separated by $\sim 400 \mathrm{pc}$, when blurred together the complex has a core diameter of $\sim 2 \mathrm{kpc}$. This 
$[\mathrm{t}]$

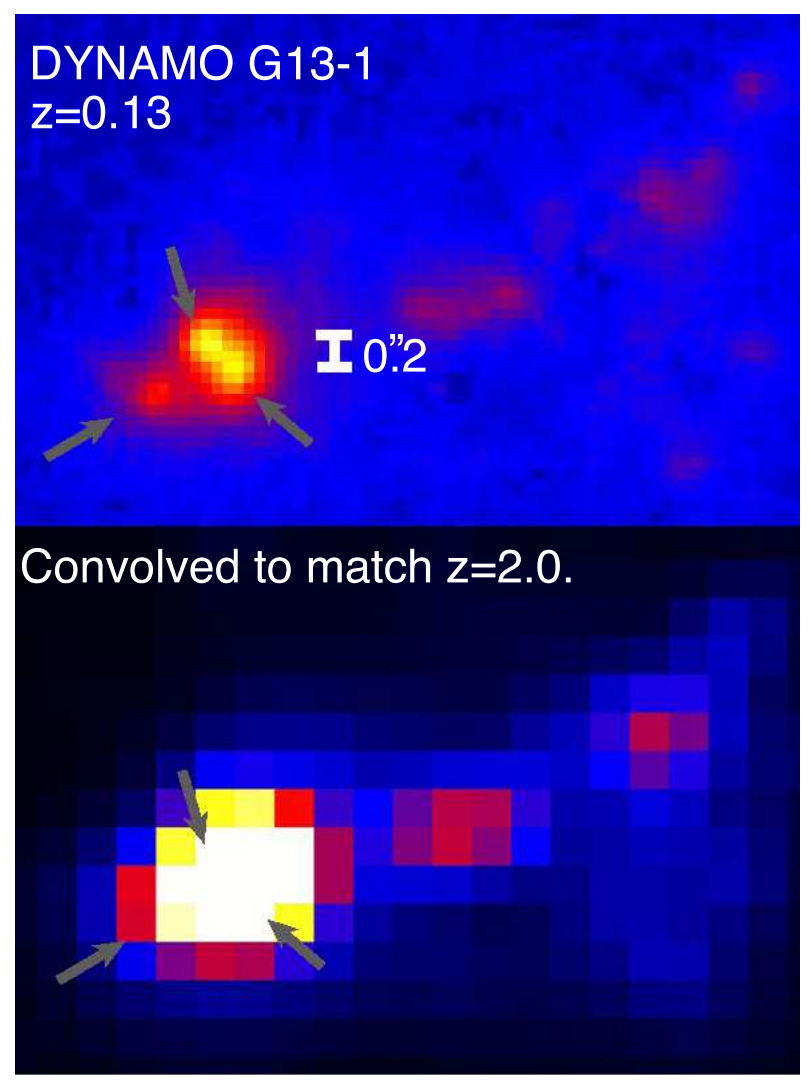

Figure 8. Top: DYNAMO galaxy G13-1 at the full resolution of $H S T$ for a $\mathrm{z}=0.13$ galaxy. Bottom: The same galaxy, but the map has been blurred to simulate the typical resolution on $\mathrm{AO}$ enhanced (or $H S T$ ) maps of $z=2$ galaxies (FWHM $1.8 \mathrm{kpc}$ ). The white bar represents $500 \mathrm{pc}$. The arrows indicate the location of 3 clumps identified in the full resolution $\mathrm{H} \alpha+[\mathrm{NII}]$ map, these clumps are blurred to appear as one giant clump at $z \sim 2$ resolution.

behavior is common in the maps, and also has the effect of the blurred DYNAMO maps having significantly fewer clumps.

Both blurring and reduced sensitivity decrease the number of clumps. In all of the clumpy DYNAMO galaxies we detect 90 clumps at full resolution (including central clumps and excluding A 04-3 \& C 13-1), roughly 11 clumps per galaxy. In the blurred maps of the same set of galaxies we detect only 35 clumps, fewer than 5 clumps per galaxy. Resolution effects alone account for a decrease in the number of clumps by a factor of $\sim 2-3$. When we account for the $(1+z)^{4}$ cosmological dimming and reduced sensitivity for restframe $\mathrm{H} \alpha$ surveys of $z \sim 1-3$ galaxies that use near-IR spectroscopy the affect on clump numbers decreases to $\sim 1-4$ per galaxy. This smaller rate of clumps in the blurred maps with a high- $z$ mimicking sensitivity degredation is comparable to the amount of clumps detected in high- $z$ surveys of unlensed galaxies (Genzel et al. 2011; Wisnioski et al. 2012; Swinbank et al. 2012).

When a blurred clump is associated with multiple clumps in the full resolution map we use the average of the associated clump properties for comparison. There is very

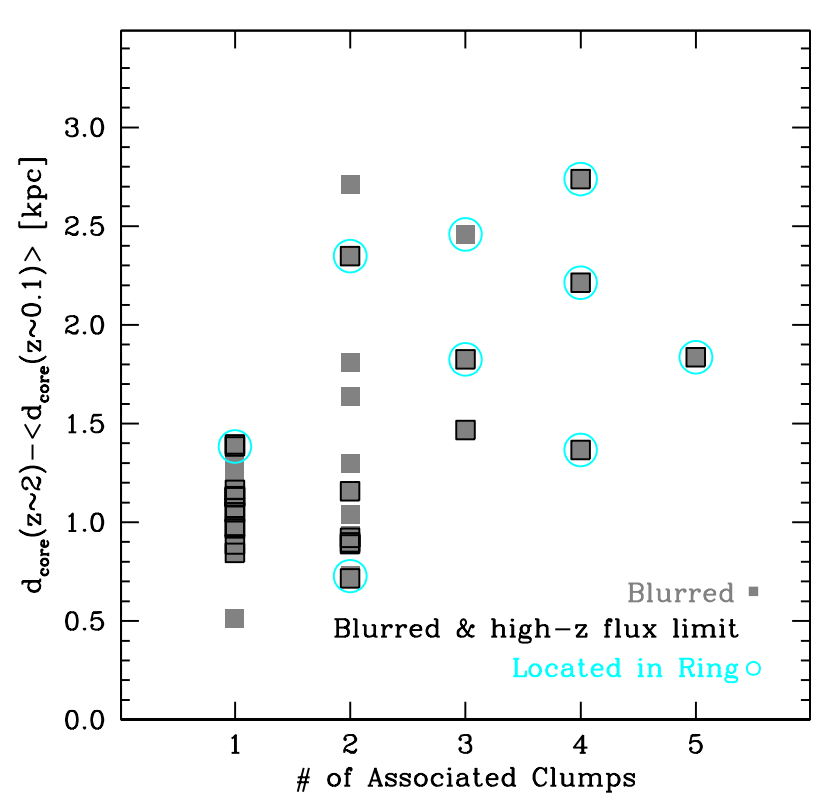

Figure 9. Here we compare the difference in core sizes from the blurred to the unblurred maps to the number of clumps in the full resolution map that are associated with a clump in the blurred maps. Grey squares indicate clumps identified in blurred $\mathrm{H} \alpha$ maps. Black open squares indicate those clumps that would be observed with the SINS sensitivity cut. Cyan rings indicate thows clumps that reside in the ring region of the disk. Clumps which correspond to a single clump on average reflect the difference in the resolution between the two measurements. However, when clumps in the blurred maps correspond to more clumps they are systematically larger.

little difference if we choose the maximum clump size or the average clump size.

In Fig. 9 we compare the difference in $d_{\text {core }}$ from blurredto-full resolution to the number of full resolution clumps corresponding to each blurred clump. When blurred clumps correspond to a single clump the difference reflects, on average, the difference between the resolution elements. As the number of corresponding full resolution clumps increases as does the affect on the measured size of the blurred clump. Blurred clumps with very large sizes $\left(d_{\text {core }} \gtrsim 2 \mathrm{kpc}\right)$ are associated with multiple clumps at full resolution in all cases.

The star formation rate of individual blurred clumps are on average higher than associated full resolution clumps by $2-3 \times$ (Fig. 10), and in some cases the star formation rate can be increased as much as $\sim 6 \times$ the star formation rate of the average full resolution clump. The increase in star formation rate is larger for blurred clumps that are associated with more than one clump. These are plausible results, since the blurred clump luminosity should be very close the sum of the full resolution clumps, ie for a blurred clump $L_{\text {blurred }} \sim \Sigma L_{\text {full }} \sim N_{\text {clump }}<L_{\text {full }}>\sim 2-5<L_{\text {full }}>$. The largest enhancements to the blurred clump star formation rate occur in those clumps located in rings, again where clumps are more crowded and emission from the ring can also be included in the blurred measurement.

For blurred DYNAMO clumps the surface brightness can be as much as $20 \times$ fainter than when measured on the 

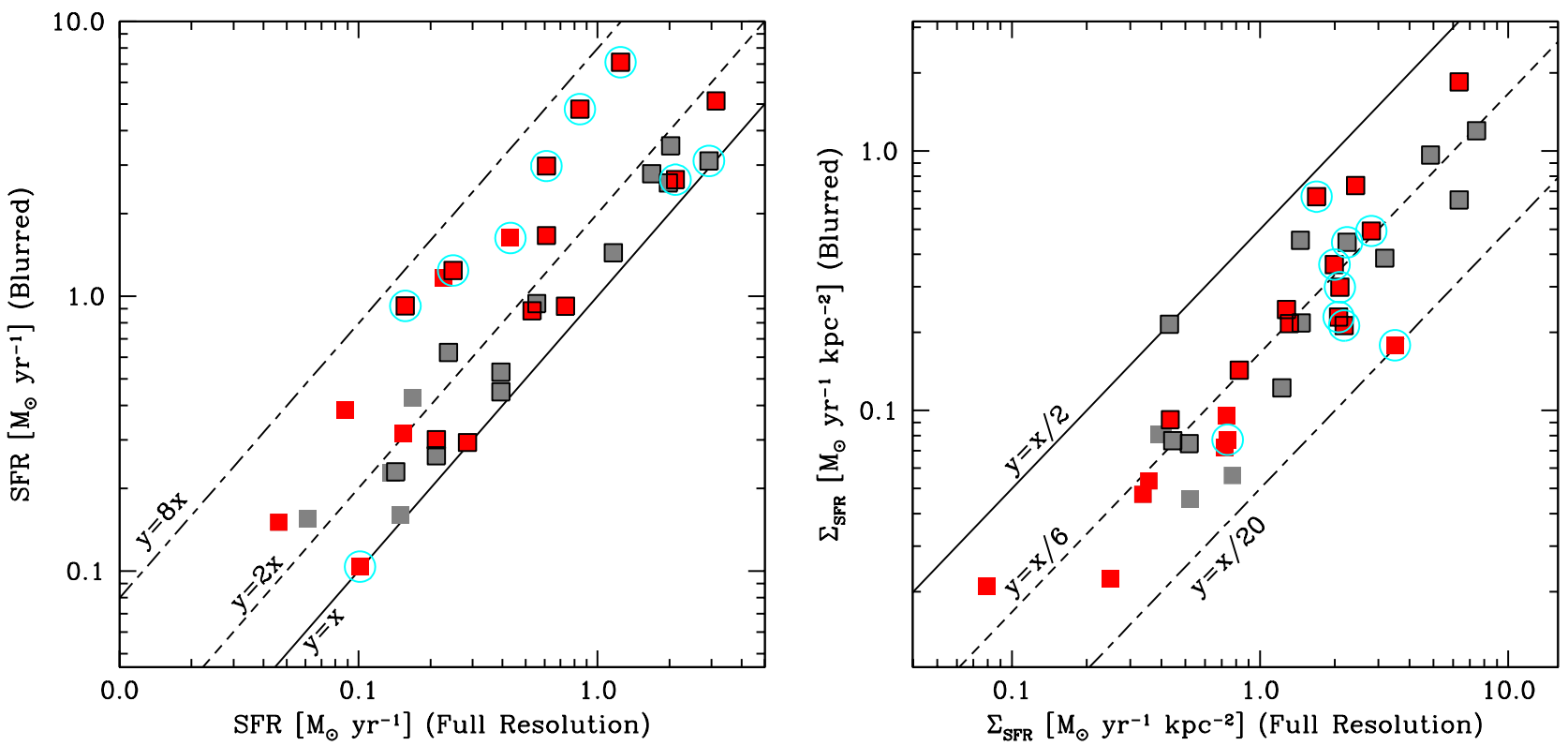

Figure 10. Here we show how degrading images to match $z \sim 2$ conditions affects the physical properties of individual clumps. The grey squares indicate blurred clumps which correspond to a single high resolution clump. Red squares indicate blurred clumps that correspond to more than one clump in the high resolution map (as illustrated in Fig. 8). The lines indicate scale factor differences between the two quantities. In the left panel we show the impact of clump clustering on the derived SFR of blurred clumps in the DYNAMO sample. When blurred clumps correspond to more than one clump in the full resolution map and/or when blurred clumps reside in rings the impact of lower resolution on luminosity is more severe. In the right panel we show how blurring of clumps affects the measured SFR surface density of clumps. The SFR surface density of blurred clumps ranges from $1 / 2$ to $1 / 20$ the surface brightness of clumps at full resolution.

full resolution maps (right panel of Fig. 10). The typical effect on $\Sigma_{S F R}$ is to decrease the surface brightness by roughly a factor of $6 \times$ the average surface brightness of corresponding clumps in the full resolution map.

In short our data suggests the clustered nature of clumps appears to exacerbate the effects of resolution degradation on clump measurements in blurred DYNAMO maps. Clump clustering enhances the measured size of clumps significantly more than simply the blurring due to the larger physical size of the beam.

\section{THE STAR FORMATION RATE SURFACE DENSITY OF GIANT CLUMPS COMPARED TO STAR FORMING REGIONS IN NEARBY GALAXIES}

In massive nearby disk galaxies the star formation rate surface density ranges between $10^{-4}-10^{-1} \mathrm{M}_{\odot} \mathrm{yr}^{-1} \mathrm{kpc}^{-2}$ (e.g. Bigiel et al. 2008; Leroy et al. 2008; Rahman et al. 2012). At $z \sim 0$ very high star formation rate surface densities are only found in extreme environments such as advanced stage mergers (Sanders \& Mirabel 1996) or disk galaxy centers with high stellar surface densities (Fisher et al. 2013). However we report that the star formation rate surface density of DYNAMO clumps in the main body of a set of rotating, exponential disks that is an order of magnitude higher than what is typical of star forming regions in disk galaxies. This is consistent with the star formation rate surface density of clumps in lensed galaxies (Livermore et al. 2012). In Fig. 11 we show that there is very little overlap between the star formation rate surface density of clumps and that of star forming regions in nearby spirals.

The vertical line in the left panel of Fig. 11 represents an H $\alpha$ clump that is $8 \%$ of the total light of the galaxy. There is a strong correlation with the surface density of the star forming regions and the clumpiness of the galaxy. Combining the sample of clumpy DYNAMO galaxies and LBA galaxies we find that in the same sample only 7 of 103 clumps have $\Sigma_{S F R}<0.5 \mathrm{M}_{\odot} \mathrm{yr}^{-1} \mathrm{kpc}^{-2}$. Conversely in the sample of more modestly star forming spiral galaxies (M 83, M 51, C 13-1 and A 04-3) we find that no star forming region in these galaxies is brighter than $L_{\text {clump }} / L_{\text {total }}>8 \%$, and only 5 out of 101 clumps have $\Sigma_{S F R}>0.5 \mathrm{M}_{\odot} \mathrm{yr}^{-1} \mathrm{kpc}^{-2}$. Using a dividing line of $\Sigma_{S F R}=0.5 \mathrm{M}_{\odot} \mathrm{yr}^{-1} \mathrm{kpc}^{-2}$ to identify giant clumps is therefore accurate to $95 \%$.

In the right panel of Fig. 11 we also compare the clump size $\left(d_{\text {core }}\right)$ to the star formation rate surface density. DYNAMO clumps are not systematically smaller than star forming regions in nearby disks. In fact the opposite is true, DYNAMO clumps on average are larger, with significant overlap.

Similar to local disks we also measure clump properties in The Antenna advanced stage merger system. None of the clumps in the Antennae are larger than the $8 \%$ luminosity threshold set by the nearby disks, nonetheless the Antennae system has high $\Sigma_{S F R}$ (median $0.6 \quad \mathrm{M}_{\odot} \mathrm{yr}^{-1} \mathrm{kpc}^{-2}$ ). The distribution of $\Sigma_{S F R}$ in the Antennae system bridges the divide between local spirals and turbulent disks. The 


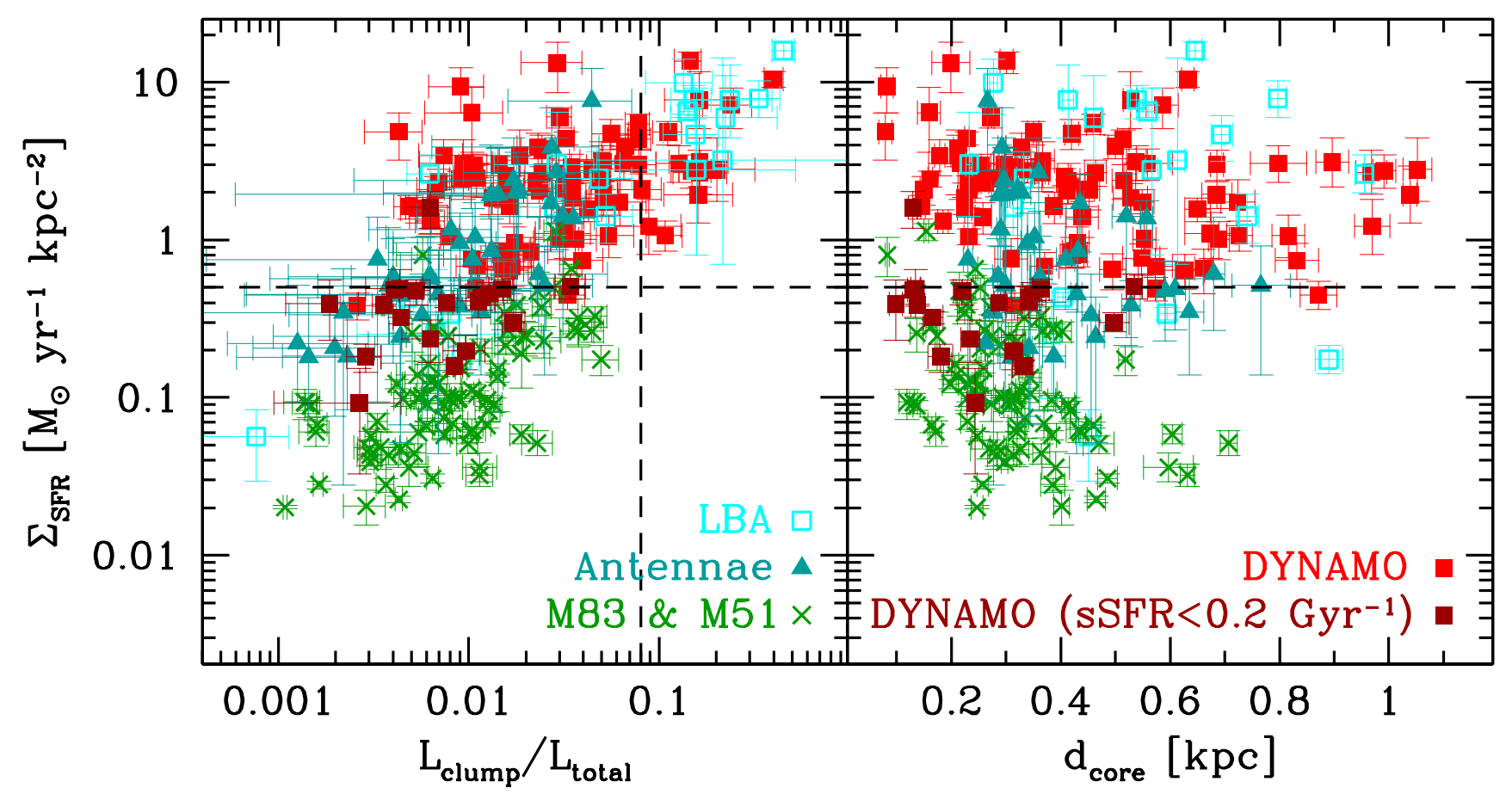

Figure 11. Here we compare the surface density of clumps to the clump-to-total H $\alpha$ ratio (left) and the core diameter of clumps (right panel). Comparison of clump properties of DYNAMO clumps to clumps in nearby star forming disks M83 \& M51, and the Antenna advanced-stage merging system. Note that the images for, Antenna, M 83 and M 51 have been blurred to match the resolution of a galaxy observed at $z=0.1$ with ACS/WFC. Symbol colors and shapes are as described in the key. Symbols for DYNAMO clumps are same as described in Fig. 7. In our data set the star formation rate surface density provides a single paramter that can clearly distinguish clumps in galaxies like DYNAMO and LBA systems, to those in more typical $z \sim 0$ galaxies like A04-3, M 51 and M 83.

average core diameter of Antennae clumps is $400 \mathrm{pc}$, similar size to those in DYNAMO galaxies. The clumps in DYNAMO mergers are somewhat larger than in clumpy disks, in DYNAMO mergers $<d_{\text {core }}>\sim 0.6 \mathrm{kpc}$, compared to $0.4 \mathrm{kpc}$ for clumps in clumpy disks. There is, however, almost no difference in the typical clump star formation rate surface density in DYNAMO mergers compared to those in disks $<\Sigma_{S F R}>\sim 2.5 \mathrm{M}_{\odot} \mathrm{yr}^{-1} \mathrm{kpc}^{-2}$ in DYNAMO mergers, compared to $2.9 \mathrm{M}_{\odot} \mathrm{yr}^{-1} \mathrm{kpc}^{-2}$ in clumpy DYNAMO disks. We do not find any significant difference in the relationship between the size and luminosity of clumps in DYNAMO mergers vs those in clumpy disks.

In short, it appears that $\Sigma_{S F R}$ may provide a simple, single parameter that can distinguish the star formation in massive star forming clumps from that of star forming regions more commonly found in $z \sim 0$ disk galaxies. Combining $\Sigma_{S F R}$ with the maximum clump-to-total flux ratio further seperates all systems that are more typical of the low redshift Universe (Antenna, M83, and M51) from those that are interpretted as being more similar to galaxies of the high- $z$ Universe (DYNAMO and LBAs).

\section{SUMMARY \& DISCUSSION OF RESULTS}

We have analyzed the properties of $\mathrm{H} \alpha$ emitting regions in a sample of galaxies that are very well matched in properties to $z \sim 1$ main sequence galaxies.

\subsection{Similarity of DYNAMO to high-z clumpy galaxies}

When blurred to match the resolution of a galaxy at $z \sim 2$ DYNAMO galaxies appear indistinguishable from galaxies observed in high redshift surveys (eg. Genzel et al. 2011; Wisnioski et al. 2012; Swinbank et al. 2012). In blurred maps, 8 of 10 DYNAMO galaxies satisfy the criteria from Guo et al. (2015) for a "clumpy" galaxy (that being at least one clump contains $8 \%$ of the light, and is not co-located with the galaxy center). Clumps in these blurred DYNAMO maps have a similar distribution of both size and luminosity relative to the host galaxy to those in $z \sim 1-2$ galaxies (Fig. 5). We therefore conclude that DYNAMO galaxies are indeed "clumpy" galaxies. We find that DYNAMO galaxies with increasing $\sigma / V$ and specific star formation rate contain clumps that are a larger fraction of the total $\mathrm{H} \alpha$ luminosity of the galaxy.

This result is consistent with results from Green et al. (2013) and Fisher et al. (2014) that DYNAMO galaxies have rotating kinematics with high internal velocity dispersions $\left(\sigma_{H \alpha} \sim 30-60 \mathrm{~km} \mathrm{~s}^{-1}\right)$, and are rich in molecular gas $\left(f_{\text {gas }} \sim 30 \%\right)$. Recently Obreschkow et al. (2015) show that DYNAMO disk galaxies are significantly lower angular momentum systems than more typical $z \sim 0$ disks, which they argue corresponds to the expectation for similar mass galaxies at $z \sim 2$. Thus it appears that in many critical ways DYNAMO galaxies are very similar to $z \sim 1-2$ main-sequence disk galaxies. A straightforward interpretation of these re- 
sults is that if galaxy is extremely gas rich disk and that gas is turbulent, then independent of the galaxy's redshift the internal physics of gas and subsequent star formation remains very similar.

\subsection{Effect of clump clustering on clump properties measured at $\sim 1 \mathrm{kpc}$ resolution}

When studied at $100 \mathrm{pc}$ resolution, the sizes of DYNAMO clumps are similar to those in lensed galaxies (Jones et al. 2010; Livermore et al. 2012, 2015) as well as star forming regions in LBAs (Fig. 7 and Overzier et al. 2009). In all studies to date of gas rich and/or turbulent disk galaxies that have resolution below $\sim 200$ pc the size of clumps is found to be a few hundred parsecs.

We find, using blurred DYNAMO maps, that the combination of the both poorer resolution and the spatial distribution of clumps in the galaxy exacerbates the effect on the properties of clumps when viewed with FWHM 1-2 kpc. As we illustrate in Fig. 8 clumps that are clustered together in high resolution DYNAMO $H S T \mathrm{H} \alpha$ maps appear as a single much larger clump in maps that have been blurred to match $z \sim 2$ resolution.

In the DYNAMO sample clump clustering affects the basic properties of clumps in systematic ways. The systematic affects on clump properties are shown in Figs. 9, and 10 .

- Size of clumps is increased more than simply the size of the beam. We find that excessively large clumps $\left(d_{\text {core }} \sim 2-3 \mathrm{kpc}\right)$ in blurred DYNAMO maps correspond to larger numbers of DYNAMO clumps in the nominal $z \sim 0.1$ resolution maps.

- Number of clumps are significantly reduced: When multiple clumps are clustered together in a higher resolution map and then imaged at lower resolution those clumps are counted as a single clump. Clump cluster systematically acts to reduce measured number of clumps from $\sim 7-12$ clumps per galaxy to $\sim 1-3$ clumps per galaxy at the lower (FWHM $\sim 1-2 \mathrm{kpc})$ resolution.

- Clump clustering increases star formation rate: On average the increase in star formation rate due to resolution is found to be a factor of a few. Some studies assume that resolution generates an order of magnitude difference in the baryonic mass (e.g. Tamburello et al. 2015). These studies cite the difference in clump masses in measured lensed galaxies from those in unlensed galaxies as evidence for this decrease. However the total mass or star formation rate of the targeted galaxy must be considered. As we show in Fig. 1, lensed samples on average target lower mass and lower star formation rate galaxies compared to both DYNAMO and samples of unlensed high- $z$ clumpy galaxies. If we assume that the mass of the clump is mainly gas and that the gas mass scales with the star formation rate, then our results suggest such a large difference is possible but not the rule. This result is consistent with a straightforward logic: if a few clumps are clustered together the blurred clump will be the sum of the flux from the clumps plus any lower emission flux that surrounds them. Since the clumps are by definition brighter than the surrounding region, the increase in flux will be $L_{\text {blur }} /<L_{\text {resolved }}>\sim N_{\text {clumps }}$. It is very unlikely that this effect could lead to an order of magnitude increase in flux or thus mass of blurred clumps.

We find that clumps located in rings are most affected by the loss in resolution, creating a typical increase in blurred clump star formation rate of $\sim 5 \times$ that of the unblurred clumps. This is likely because both clumps are clustered more in rings, and the $\mathrm{H} \alpha$ emission in the ring likely is combined into the blurred clump flux.

- Clump star formation rate surface density is strongly affected by resolution The larger sizes of blurred clumps creates systematically lower star formation rate surface densities by as much as an order of magnitude, and also significantly fewer numbers of clumps per galaxy (Fig. 10). The star formation rate surface density of full resolution clump measurements ranges $2-20 \times$, and is typically a factor of $6 \times$ that of the blurred clump. If we consider the implication of this on the results of high-redshift galaxies, where impressivelly high star formation rate surface densities for clumps of $1-10 \mathrm{M}_{\odot} \mathrm{yr}^{-1} \mathrm{kpc}^{-2}$ are reported with a beam FWHM 1.5-2 kpc (Genzel et al. 2011), an average factor of $6 \times$ increase to these star formation rate surface densities the properties are even more extreme.

Meurer et al. (1997) find that the star burst intensity of UV bright galaxies is limited, as indicated by the absence of galaxies with greater than $\Sigma_{S F R}($ limit $) \sim 25 \mathrm{M}_{\odot} \mathrm{yr}^{-1} \mathrm{kpc}^{-2}$. The implication of such a limit is that some physical mechanism exists which regulates the star formation, and this mechanism is Universal. The derived $\Sigma_{S F R}$ of clumps in the samples with study is consistent with this limit (Genzel et al. 2011). However, if these measured $\Sigma_{S F R}$ are artificially low due to resolution effects, then clumps in $z \sim 1-2$ main-sequence galaxies may violate this limit, similar to the handful of super-intense starburst in observed in extreme galaxies (e.g. Swinbank et al. 2010; Hodge et al. 2015).

\subsection{Star formation rate surface density of clumps in turbulent disks}

We show in Fig. 11 that the star formation rate surface density of DYNAMO clumps is $\Sigma_{S F R} \sim 1-10 \mathrm{M}_{\odot} \mathrm{yr}^{-1} \mathrm{kpc}^{-2}$. This is orders of magnitude higher than what is found in the star forming regions of nearby spiral galaxies (e.g. Bigiel et al. 2008; Leroy et al. 2008; Rahman et al. 2012). In the local Universe the only other place to find such high star formation rate surface densities are shocks in galaxy-galaxy mergers (Wei et al. 2012; Zaragoza-Cardiel et al. 2014) or galaxy centers (Fisher et al. 2013).

In Fig. 11 we show that the star formation rate surface density offers a simple, physically motivated method to distinguish massive star forming clumps, characteristic of star formation in massive $z \sim 1-2$ disk galaxies, from star formation that is more typical of low gas fraction disks of the nearby Universe. The dividing line of $\Sigma_{S F R} \sim$ $0.5 \mathrm{M}_{\odot} \mathrm{yr}^{-1} \mathrm{kpc}^{-2}$ is shown to be a good way to distinguish massive star forming clumps from more typical star forming regions of $z \sim 0$ spiral galaxies.

Following arguments from the Larson (1981) relations (Elmegreen 1989, also see arguements in) the pressure increases in molecular clouds as the square of the surface density of the molecular gas, $P_{\text {ext }} \propto \Sigma_{\text {mol }}^{2}$. Clumps in DYNAMO galaxies are shown to have $\Sigma_{S F R}$ that are $\sim 500 \times$ 
that of star forming regions in nearby spirals. It is likely that the gas mass surface density is similarly high in clumps. We convert the star formation rate surface density to gas mass surface density using the global scaling for DYNAMO disks in Fisher et al. (2014) of find $M_{m o l} / S F R \sim 5 \times 10^{8} \mathrm{yr}$. The pressure in the massive star forming clumps in a DYNAMO galaxy is therefore $10^{4} \times$ that found in star forming regions of nearby spirals. These high pressures are only observed in the most extreme clouds in the center of the Milky Way (Rathborne et al. 2014), and high- $z$ galaxies (Swinbank et al. 2010, 2015). It remains unclear if the conditions in these environments are consistent with models of star formation (eg. Krumholz \& McKee 2005). These systems therefore warrant more work with facilities such as ALMA to accurately describe the star formation efficiency, and ratios of dense gas gas inside the star forming regions, and also to understand what drives the turbulent gas that creates these clumps.

In this work we have again shown that the DYNAMO sample is an excellent laboratory to study the conditions of galaxies at $z \sim 1-3$, with which they are consistent. In future work we will compare expectations from the violent disk instability model to the clump properties of DYNAMO galaxies (Fisher et al. in prep), investigate systematic correlations fo DYNAMO dust and gas properties in galaxies (Bassett et al. submitted, White et al. in prep), and study the properties of clumps in stellar continuum (Fisher et al. in prep).

\section{ACKNOWLEDGEMENTS}

DBF acknolwedges support from Australian Research Council (ARC) Discovery Program (DP) grant DP130101460. Support for this project is provided in part by the Victorian Department of State Development, Business and Innovation through the Victorian International Research Scholarship (VIRS).

\section{REFERENCES}

Abraham R. G., Tanvir N. R., Santiago B. X., Ellis R. S., Glazebrook K., van den Bergh S., 1996, MNRAS, 279, L47

Adelman-McCarthy J. K., et al. 2006, ApJS, 162, 38

Bassett R., et al., 2014, MNRAS, 442, 3206

Basu-Zych A. R., et al., 2009, ApJ, 699, L118

Behrendt M., Burkert A., Schartmann M., 2015, preprint, (arXiv: 1512.03430)

Bekiaris G., Glazebrook K., Fluke C. J., Abraham R., 2015, preprint, (arXiv: 1510.01815)

Bigiel F., Leroy A., Walter F., Brinks E., de Blok W. J. G., Madore B., Thornley M. D., 2008, AJ, 136, 2846

Bournaud F., et al., 2014, ApJ, 780, 57

Brandl B. R., et al., 2009, ApJ, 699, 1982

Brinchmann J., Charlot S., White S. D. M., Tremonti C., Kauffmann G., Heckman T., Brinkmann J., 2004, MNRAS, 351,1151

Calvi V., Stiavelli M., Bradley L., Pizzella A., Kim S., 2014, ApJ, 796, 102

Conselice C. J., Bershady M. A., Jangren A., 2000, ApJ, 529, 886

Daddi E., et al., 2010, ApJ, 713, 686

Dekel A., Sari R., Ceverino D., 2009, ApJ, 703, 785
Elmegreen B. G., 1989, ApJ, 344, 306

Elmegreen B. G., Elmegreen D. M., 2005, ApJ, 627, 632

Elmegreen D. M., Elmegreen B. G., Rubin D. S., Schaffer M. A., 2005, ApJ, 631, 85

Epinat B., et al., 2009, A\&A, 504, 789

Fisher D. B., Drory N., 2008, AJ, 136, 773

Fisher D. B., Bolatto A., Drory N., Combes F., Blitz L., Wong T., 2013, ApJ, 764, 174

Fisher D. B., et al., 2014, ApJ, 790, L30

Förster Schreiber N. M., et al., 2009, ApJ, 706, 1364

Förster Schreiber N. M., Shapley A. E., Erb D. K., Genzel R., Steidel C. C., Bouché N., Cresci G., Davies R., 2011a, ApJ, 731,65

Förster Schreiber N. M., et al., 2011b, ApJ, 739, 45

Genzel R., et al., 2011, ApJ, 733, 101

Gonçalves T. S., et al., 2010, ApJ, 724, 1373

Green A. W., et al., 2010, Nature, 467, 684

Green A. W., et al., 2013, MNRAS,

Green A. W., et al., 2014, MNRAS, 437, 1070

Guo Y., et al., 2015, ApJ, 800, 39

Hao C.-N., Kennicutt R. C., Johnson B. D., Calzetti D., Dale D. A., Moustakas J., 2011, ApJ, 741, 124

Heckman T. M., et al., 2005, ApJ, 619, L35

Heckman T. M., et al., 2011, ApJ, 730, 5

Hodge J. A., Carilli C. L., Walter F., de Blok W. J. G., Riechers D., Daddi E., Lentati L., 2012, ApJ, 760, 11

Hodge J. A., Riechers D., Decarli R., Walter F., Carilli C. L., Daddi E., Dannerbauer H., 2015, ApJ, 798, L18

Hopkins A. M., Beacom J. F., 2006, ApJ, 651, 142

Jones T. A., Swinbank A. M., Ellis R. S., Richard J., Stark D. P., 2010, MNRAS, 404, 1247

Karim A., et al., 2011, ApJ, 730, 61

Kauffmann G., et al., 2003, MNRAS, 341, 54

Kennicutt Jr. R. C., 1979, ApJ, 228, 696

Krumholz M. R., McKee C. F., 2005, ApJ, 630, 250

Larson R. B., 1981, MNRAS, 194, 809

Law D. R., Steidel C. C., Erb D. K., Larkin J. E., Pettini M., Shapley A. E., Wright S. A., 2009, ApJ, 697, 2057

Leroy A. K., Walter F., Brinks E., Bigiel F., de Blok W. J. G., Madore B., Thornley M. D., 2008, AJ, 136, 2782

Livermore R. C., et al., 2012, MNRAS, 427, 688

Livermore R. C., et al., 2015, MNRAS, 450, 1812

Madau P., Dickinson M., 2014, ARA\&A, 52, 415

Meurer G. R., Heckman T. M., Lehnert M. D., Leitherer C., Lowenthal J., 1997, AJ, 114, 54

Obreschkow D., et al., 2015, preprint, (arXiv:1508.04768)

Östlin G., Hayes M., Kunth D., Mas-Hesse J. M., Leitherer C., Petrosian A., Atek H., 2009, AJ, 138, 923

Overzier R. A., et al., 2008, ApJ, 677, 37

Overzier R. A., et al., 2009, ApJ, 706, 203

Rahman N., et al., 2012, ApJ, 745, 183

Rathborne J. M., et al., 2014, ApJ, 795, L25

Robertson B., Bullock J. S., Cox T. J., Di Matteo T., Hernquist L., Springel V., Yoshida N., 2006, ApJ, 645, 986

Sanders D. B., Mirabel I. F., 1996, ARA\&A, 34, 749

Shibuya T., Ouchi M., Kubo M., Harikane Y., 2015, preprint, (arXiv:1511.07054)

Swinbank A. M., et al., 2009, MNRAS, 400, 1121

Swinbank A. M., et al., 2010, Nature, 464, 733

Swinbank A. M., Smail I., Sobral D., Theuns T., Best P. N., Geach J. E., 2012, ApJ, 760, 130

Swinbank M., et al., 2015, preprint, (arXiv:1505.05148)

Tacconi L. J., et al., 2013, ApJ, 768, 74

Tamburello V., Mayer L., Shen S., Wadsley J., 2015, MNRAS, 453,2490

Tremonti C. A., et al., 2004, ApJ, 613, 898

Wei L. H., Keto E., Ho L. C., 2012, ApJ, 750, 136 


\begin{tabular}{|c|c|c|c|}
\hline Galaxy & $\begin{array}{l}d_{\text {core }} \\
(\mathrm{pc})\end{array}$ & $\begin{array}{c}L_{H \alpha} \\
\left(10^{40} \mathrm{erg} \mathrm{s}^{-1}\right)\end{array}$ & $\begin{array}{c}\mathrm{SFR} \\
\left(M_{\odot} \mathrm{yr}^{-1}\right)\end{array}$ \\
\hline G20-2 & $547 \pm 34$ & $4.95 \pm 1.67$ & 0.27 \\
\hline G20-2 & $968 \pm 28$ & $54.03 \pm 42.98$ & 2.99 \\
\hline G20-2 & $896 \pm 30$ & $53.68 \pm 37.63$ & 2.97 \\
\hline G20-2 & $159 \pm 21$ & $3.43 \pm 1.50$ & 0.19 \\
\hline G20-2 & $1052 \pm 26$ & $66.19 \pm 61.92$ & 3.66 \\
\hline G20-2 & $798 \pm 51$ & $41.93 \pm 23.83$ & 2.32 \\
\hline G20-2 & $305 \pm 9$ & $18.16 \pm 5.39$ & 1.00 \\
\hline G20-2 & $217 \pm 59$ & $3.09 \pm 1.31$ & 0.17 \\
\hline G20-2 & $674 \pm 47$ & $10.66 \pm 5.13$ & 0.59 \\
\hline G20-2 & $650 \pm 23$ & $13.99 \pm 5.54$ & 0.77 \\
\hline G20-2 & $626 \pm 37$ & $5.39 \pm 2.16$ & 0.30 \\
\hline G13-1 & $586 \pm 29$ & $52.82 \pm 19.33$ & 2.92 \\
\hline G13-1 & $632 \pm 12$ & $88.60 \pm 26.35$ & 4.90 \\
\hline G13-1 & $555 \pm 18$ & $12.63 \pm 3.74$ & 0.70 \\
\hline G13-1 & $549 \pm 21$ & $11.30 \pm 3.21$ & 0.62 \\
\hline G13-1 & $871 \pm 34$ & $7.29 \pm 3.85$ & 0.40 \\
\hline G13-1 & $430 \pm 22$ & $3.84 \pm 1.08$ & 0.21 \\
\hline G13-1 & $553 \pm 21$ & $6.66 \pm 2.19$ & 0.37 \\
\hline G13-1 & $495 \pm 24$ & $3.41 \pm 1.06$ & 0.19 \\
\hline G13-1 & $431 \pm 17$ & $3.25 \pm 0.71$ & 0.18 \\
\hline G14-1 & $727 \pm 23$ & $12.10 \pm 4.52$ & 0.67 \\
\hline G14-1 & $407 \pm 8$ & $38.34 \pm 6.45$ & 2.12 \\
\hline G14-1 & $452 \pm 19$ & $9.18 \pm 2.60$ & 0.51 \\
\hline G14-1 & $350 \pm 10$ & $12.56 \pm 2.43$ & 0.69 \\
\hline G08-5 & $406 \pm 12$ & $8.99 \pm 1.54$ & 0.50 \\
\hline G08-5 & $301 \pm 12$ & $26.27 \pm 4.69$ & 1.45 \\
\hline G08-5 & $404 \pm 19$ & $18.64 \pm 4.31$ & 1.03 \\
\hline G08-5 & $367 \pm 21$ & $9.04 \pm 3.07$ & 0.50 \\
\hline G08-5 & $212 \pm 13$ & $3.35 \pm 1.48$ & 0.19 \\
\hline G08-5 & $421 \pm 21$ & $2.99 \pm 0.62$ & 0.17 \\
\hline G08-5 & $414 \pm 22$ & $8.78 \pm 2.71$ & 0.49 \\
\hline G08-5 & $318 \pm 21$ & $6.28 \pm 1.39$ & 0.35 \\
\hline G08-5 & $371 \pm 26$ & $2.00 \pm 0.49$ & 0.11 \\
\hline G08-5 & $439 \pm 29$ & $5.77 \pm 1.67$ & 0.32 \\
\hline $\mathrm{H} 10-2$ & $831 \pm 40$ & $11.05 \pm 6.58$ & 0.61 \\
\hline H10-2 & $573 \pm 31$ & $4.76 \pm 1.82$ & 0.26 \\
\hline H10-2 & $1039 \pm 13$ & $44.94 \pm 39.62$ & 2.49 \\
\hline H10-2 & $527 \pm 7$ & $45.37 \pm 13.53$ & 2.51 \\
\hline $\mathrm{H} 10-2$ & $445 \pm 16$ & $8.81 \pm 2.55$ & 0.49 \\
\hline H10-2 & $969 \pm 32$ & $24.71 \pm 19.39$ & 1.37 \\
\hline H10-2 & $816 \pm 30$ & $15.24 \pm 8.94$ & 0.84 \\
\hline H10-2 & $689 \pm 19$ & $10.14 \pm 4.70$ & 0.56 \\
\hline G04-1 & $233 \pm 17$ & $2.68 \pm 0.57$ & 0.15 \\
\hline G04-1 & $254 \pm 19$ & $4.10 \pm 0.76$ & 0.23 \\
\hline G04-1 & $661 \pm 17$ & $6.23 \pm 2.43$ & 0.34 \\
\hline G04-1 & $284 \pm 14$ & $4.23 \pm 1.08$ & 0.23 \\
\hline G04-1 & $538 \pm 18$ & $19.50 \pm 5.81$ & 1.08 \\
\hline G04-1 & $685 \pm 27$ & $19.50 \pm 6.35$ & 1.08 \\
\hline G04-1 & $992 \pm 21$ & $57.25 \pm 31.38$ & 3.17 \\
\hline G04-1 & $686 \pm 12$ & $30.23 \pm 10.24$ & 1.67 \\
\hline G04-1 & $297 \pm 19$ & $5.58 \pm 1.59$ & 0.31 \\
\hline G04-1 & $199 \pm 35$ & $11.27 \pm 4.11$ & 0.62 \\
\hline G04-1 & $464 \pm 20$ & $12.22 \pm 2.95$ & 0.68 \\
\hline G04-1 & $328 \pm 28$ & $8.90 \pm 2.21$ & 0.49 \\
\hline G04-1 & $515 \pm 22$ & $13.44 \pm 3.98$ & 0.74 \\
\hline
\end{tabular}

Whitaker K. E., van Dokkum P. G., Brammer G., Franx M., 2012, ApJ, 754, L29

Whitmore B. C., et al., 2010, AJ, 140, 75

Wisnioski E., et al., 2011, MNRAS, 417, 2601

Wisnioski E., Glazebrook K., Blake C., Poole G. B., Green A. W., Wyder T., Martin C., 2012, MNRAS, 422, 3339

Wuyts S., et al., 2012, ApJ, 753, 114
Wuyts S., et al., 2013, ApJ, 779, 135

Zaragoza-Cardiel J., Font J., Beckman J. E., García-Lorenzo B., Erroz-Ferrer S., Gutiérrez L., 2014, MNRAS, 445, 1412 


\begin{tabular}{|c|c|c|c|}
\hline Galaxy & $\begin{array}{l}d_{\text {core }} \\
(\mathrm{pc})\end{array}$ & $\begin{array}{c}L_{H \alpha} \\
\left(10^{40} \mathrm{erg} \mathrm{s}^{-1}\right)\end{array}$ & $\begin{array}{c}\mathrm{SFR} \\
\left(M_{\odot} \mathrm{yr}^{-1}\right)\end{array}$ \\
\hline D13-5 & $573 \pm 15$ & $3.44 \pm 1.50$ & 0.19 \\
\hline D13-5 & $315 \pm 14$ & $0.82 \pm 0.19$ & 0.05 \\
\hline D13-5 & $420 \pm 13$ & $17.67 \pm 5.55$ & 0.98 \\
\hline D13-5 & $528 \pm 31$ & $11.03 \pm 4.42$ & 0.61 \\
\hline D13-5 & $514 \pm 11$ & $24.79 \pm 8.97$ & 1.37 \\
\hline D13-5 & $500 \pm 13$ & $21.04 \pm 7.55$ & 1.16 \\
\hline D13-5 & $558 \pm 46$ & $20.58 \pm 8.99$ & 1.14 \\
\hline D13-5 & $459 \pm 12$ & $24.54 \pm 7.66$ & 1.36 \\
\hline D13-5 & $724 \pm 31$ & $19.63 \pm 13.14$ & 1.09 \\
\hline D13-5 & $119 \pm 6$ & $5.39 \pm 1.00$ & 0.30 \\
\hline D13-5 & $365 \pm 13$ & $7.66 \pm 2.28$ & 0.42 \\
\hline D13-5 & $412 \pm 18$ & $7.41 \pm 2.10$ & 0.41 \\
\hline D13-5 & $303 \pm 18$ & $4.96 \pm 1.40$ & 0.27 \\
\hline D13-5 & $388 \pm 11$ & $5.21 \pm 1.56$ & 0.29 \\
\hline D13-5 & $179 \pm 10$ & $2.33 \pm 0.63$ & 0.13 \\
\hline D13-5 & $273 \pm 5$ & $9.51 \pm 1.82$ & 0.53 \\
\hline D13-5 & $258 \pm 15$ & $1.99 \pm 0.48$ & 0.11 \\
\hline D13-5 & $224 \pm 13$ & $1.70 \pm 0.35$ & 0.09 \\
\hline D15-3 & $149 \pm 7$ & $1.01 \pm 0.22$ & 0.06 \\
\hline D15-3 & $184 \pm 9$ & $0.95 \pm 0.19$ & 0.05 \\
\hline D15-3 & $82 \pm 11$ & $1.36 \pm 0.44$ & 0.08 \\
\hline D15-3 & $339 \pm 19$ & $6.73 \pm 1.94$ & 0.37 \\
\hline D15-3 & $213 \pm 9$ & $3.63 \pm 0.90$ & 0.20 \\
\hline D15-3 & $79 \pm 12$ & $0.64 \pm 0.22$ & 0.04 \\
\hline D15-3 & $224 \pm 13$ & $1.99 \pm 0.51$ & 0.11 \\
\hline D15-3 & $83 \pm 7$ & $1.31 \pm 0.35$ & 0.07 \\
\hline D15-3 & $231 \pm 13$ & $2.37 \pm 0.62$ & 0.13 \\
\hline D15-3 & $261 \pm 7$ & $3.38 \pm 0.68$ & 0.19 \\
\hline D15-3 & $309 \pm 30$ & $1.54 \pm 0.59$ & 0.09 \\
\hline D15-3 & $420 \pm 17$ & $3.18 \pm 1.30$ & 0.18 \\
\hline D15-3 & $161 \pm 4$ & $1.34 \pm 0.18$ & 0.07 \\
\hline D15-3 & $232 \pm 8$ & $1.19 \pm 0.20$ & 0.07 \\
\hline D15-3 & $228 \pm 4$ & $4.88 \pm 0.74$ & 0.27 \\
\hline D15-3 & $144 \pm 8$ & $0.73 \pm 0.14$ & 0.04 \\
\hline C13-1 & $535 \pm 14$ & $3.12 \pm 1.54$ & 0.17 \\
\hline C13-1 & $364 \pm 16$ & $1.37 \pm 0.37$ & 0.08 \\
\hline C13-1 & $288 \pm 10$ & $0.70 \pm 0.12$ & 0.04 \\
\hline C13-1 & $130 \pm 8$ & $0.58 \pm 0.15$ & 0.03 \\
\hline C13-1 & $219 \pm 16$ & $0.49 \pm 0.10$ & 0.03 \\
\hline C13-1 & $235 \pm 4$ & $5.79 \pm 0.88$ & 0.32 \\
\hline C13-1 & $348 \pm 17$ & $1.18 \pm 0.31$ & 0.07 \\
\hline C13-1 & $342 \pm 15$ & $1.06 \pm 0.28$ & 0.06 \\
\hline C13-1 & $342 \pm 31$ & $1.03 \pm 0.30$ & 0.06 \\
\hline C13-1 & $498 \pm 27$ & $1.57 \pm 0.68$ & 0.09 \\
\hline A04-3 & $234 \pm 18$ & $0.27 \pm 0.09$ & 0.02 \\
\hline A04-3 & $98 \pm 12$ & $0.08 \pm 0.03$ & 0.004 \\
\hline A04-3 & $135 \pm 9$ & $0.19 \pm 0.06$ & 0.01 \\
\hline A04-3 & $220 \pm 10$ & $1.03 \pm 0.28$ & 0.06 \\
\hline A04-3 & $244 \pm 16$ & $0.12 \pm 0.08$ & 0.01 \\
\hline A04-3 & $314 \pm 17$ & $0.42 \pm 0.15$ & 0.02 \\
\hline A04-3 & $331 \pm 19$ & $0.37 \pm 0.11$ & 0.02 \\
\hline A04-3 & $138 \pm 13$ & $0.16 \pm 0.01$ & 0.01 \\
\hline A04-3 & $180 \pm 23$ & $0.13 \pm 0.03$ & 0.01 \\
\hline A04-3 & $166 \pm 18$ & $0.19 \pm 0.03$ & 0.01 \\
\hline A04-3 & $131 \pm 11$ & $0.18 \pm 0.02$ & 0.01 \\
\hline
\end{tabular}

\section{APPENDIX A: COMPARISON SAMPLES}

\section{A1 Comparison sample 1: High-z galaxies and high-z analogues}

The DYNAMO-HST data set occupies a unique section of parameter space. The FWHM of DYNAMO-HST $H \alpha+[\mathrm{NII}]$ maps is $\sim 0.07-0.12$ arcsec, corresponding to a physical reso- lution of $\sim 150-300 \mathrm{pc}$. The physical resolution from observing DYNAMO galaxies is roughly from $\sim 3-10 \times$ finer compared to AO-enabled or HST observations of high- $z$ galaxies, similar to what is achieved from lens galaxies, however unlike lensed galaxies in DYNAMO the improvement in resolution is in both directions. As we show in Fig. 1 the SFR and stellar mass of DYNAMO galaxies is typically $\sim 10 \times$ that 
of typical lensed galaxies with clump measurements. The average clump SFR is larger in galaxies with more star formation (Livermore et al. 2012). A direct comparison of the properties of star forming clumps should control for total SFR.

Here we summarize the salient features of each of the high- $z$ comparison data sets of clump properties.

SINS: The clump measurements are taken from Genzel et al. (2011), which is a subset of the SINS survey (Förster Schreiber et al. 2009). As we show in Fig. 1, the galaxies in the Genzel et al. (2011) sample have the largest star formation rates in our comparison sample, ranging 30-200 $\mathrm{M}_{\odot} \mathrm{yr}^{-1}$. Stellar masses of this SINS subset range $10^{10}-10^{11} \mathrm{M}_{\odot}$. The galaxies in the SINS clump sample have $z \sim 2.2-2.3$. The observations of the SINS clump sample were made with SINFONI-AO, achieving typical resolution of $\sim 0.22$ arcsec, or $1.85 \mathrm{kpc}$. Genzel et al. (2011) use the width of Gaussians as a metric of clump sizes, similar to what we use.

WiggleZ: The clump properties are taken from Wisnioski et al. (2012), which is a subset of $z \sim 1.3-1.5$ galaxies in the WiggleZ Dark Energy survey. The galaxy masses and SFR $\left(5-10 \times 10^{10} \mathrm{M}_{\odot}\right.$ and $\left.10-30 \mathrm{M}_{\odot} \mathrm{yr}^{-1}\right)$ are consistent with the $z \sim 1$ main-sequence. Observations of WiggleZ clumps are made with Keck/OSIRIS$\mathrm{AO}$ and have a resolution of $\sim 0.1$ arcsec, or $0.85 \mathrm{kpc}$. Wisnioski et al. (2012) uses Gaussian RMS. We therefore adjust the Wisnioski et al. (2012) clumps sizes $R=$ $R_{\text {wis }} \sqrt{(2 \ln 2) \text {. }}$

SHiZELS: The clump measurements for the SHiZELS survey are taken from Swinbank et al. (2012). Galaxy masses span $0.6-10 \times 10^{10} \mathrm{M}_{\odot}$ and SFR span 1-30 $M_{\odot} \mathrm{yr}^{-1}$. The redshifts of 3 SHiZELS galaxies are $z \sim 1.5$, and 1 at $z \sim 2.2$. Observations of these galaxies are made with SINFONI+AO with approximate spatial resolution of 0.1 arcsec, corresponding to $0.85 \mathrm{kpc}$.

Jones + 2010: These observations are made on a sample of strongly lensed, star forming galaxies at $z \sim 1.7-3.1$. The masses range $0.5-2 \times 10^{10} \mathrm{M}_{\odot}$ and the SFR range 2$40 \mathrm{M}_{\odot} \mathrm{yr}^{-1}$. Observations are made with the Keck/OSIRIS AO system, obtaining resolution of 0.11 arcsec. The lensing magnification in these galaxies was typically $\sim 8 \times$, which results in typical resolution one direction of $\sim 0.1 \mathrm{kpc}$. Note however this magnification is only in one direction.

In this paper we determine clump sizes through a Gaussian fitting technicque (described in section 3). Jones et al. (2010) and Swinbank et al. (2012) both use "isophotal sizes". Wisnioski et al. (2012) shows that this method systematically returns larger sizes compared to the Gaussian fitting methods, but an adjustment factor is not determined. We therefore use these published sizes as is, with the caveat that in comparison to our clump sizes, these may be slightly larger.

Livermore + 2012: These observations of clumps are also made on strongly lensed galaxies in the redshift range $z \sim 0.9-1.5$. Though total masses are not given for this sample, typical SFR range $0.4-12 \mathrm{M}_{\odot} \mathrm{yr}^{-1}$. The observations are made with narrowband filters on $H S T / W F C 3$, the median resolution in one direction for the sample is $0.36 \mathrm{kpc}$.

Livermore + 2015: These observations of clumps are also made on strongly lensed galaxies in the redshift range $z \sim 1-4$. For galaxies in this sample the SFR ranges $0.8-30 \mathrm{M}_{\odot} \mathrm{yr}^{-1}$ and total stellar masses range 0.06 - $0.3 \times 10^{10} \mathrm{M}_{\odot}$. The observations are made with SINFONI IFU on the ESO/VLT, the resolution one direction for the sample spans 0.1-0.7 kpc.

The Livermore et al. (2015) sample also includes a reanalysis of the galaxies in Jones et al. (2010). In the interest of reducing systematic biases, and increases the base of our comparison we opt to use values from Jones et al. (2010).

Livermore et al. (2012) and Livermore et al. (2015) uses the sizes of clumps returned from the software CLUMPFIND. Livermore et al. (2012) shows that sizes produced from CLUMPFIND are systematically 25\% larger than Gaussian FWHM sizes (which we use in this paper); we therefore adjust the sizes from CLUMPIND by $R=R_{l i v} / 1.25$.

Lyman Break Analogs (LBA) - We also include a sample of Lyman Break Analog galaxies for comparison. These are not high-redshift galaxies, however ther have properties that are very similar to high redshift Lyman Break Galaxies (Heckman et al. 2005; Östlin et al. 2009; Gonçalves et al. 2010). We use galaxies Haro 11, IRAS08339+6517 and NGC 6090 from Heckman et al. (2011) describing LBA galaxies. The HST maps we use are presented and discussed in Östlin et al. (2009). As we discuss above, LBA systems are more likely to resemble high$z$ merging galaxies than turbulent disks. The LBA sample therefore provides a complimentary comparison to the DYNAMO sample. We degrade $\mathrm{H} \alpha$ maps of the LBA galaxies to match the resolution of a galaxy at $z \sim 0.1$ that is observed with ACS/WFC, so that there is a straightforward comparison to DYNAMO galaxies. The clump properties are derived in the same procedure as is used for the DYNAMO galaxies.

\section{A2 Comparison Sample 2: Nearby Spiral and Merging Galaxies}

As is indicated in Fig. 1, the DYNAMO-HST sample contains one galaxy that is selected to be consistent with the $z \sim 0.1$ main sequence. This galaxy, A04-3, has both a modest $\mathrm{SFR}=3.4 \mathrm{M}_{\odot} \mathrm{yr}^{-1}$ and velocity dispersion $\sigma_{H \alpha}=10 \mathrm{~km} \mathrm{~s}^{-1}$. We choose to observe this galaxy as a control for our method. We also make similar measurements to clumps properties on galaxies in the nearby Universe. We choose two well known disks and one well known merger. To make the comparison as uniform as possible we select only comparison galaxies with available $\mathrm{H} \alpha+[\mathrm{NII}]$ maps available in the HST archive. We then degrade the resolution of the image to match that of a galaxy observed at $z=0.1$ with ACS/WFC.

The comparison samples include the following galaxies: Arp 244 - The so-called Antennae galaxy is an advanced stage merger with a large set of very active star forming regions. We utilize the the map from Whitmore et al. (2010) targeting the middle, star bursting section of the galaxy. The total SFR of the Antennae system is $\sim 5-10 \mathrm{M}_{\odot} \mathrm{yr}^{-1}$ (Brandl et al. 2009), placing this galaxy at the low end of the SFRs observed in DYNAMO galaxies.

M 83 and M 51 - These galaxies are chosen to be representative of $z \sim 0$ star forming disks. The galaxies M 83 and M 51 have comparable stellar mass to DYNAMO galaxies $\left(1-3 \times 10^{10} \mathrm{M}_{\odot}\right)$, however compared to most of the galaxies in the DYNAMO-HST sample they have much more modest star formation rates $\left(\sim 1-2 \mathrm{M}_{\odot} \mathrm{yr}^{-1}\right)$, and also lower molecular gas fractions $(\sim 1-5 \%)$. 
We convolved the $\mathrm{H} \alpha$ maps of M 83, M 51 and Arp 244 with a symmetric 2-D Gaussian to simulate the resolution they would have if these well known, nearby galaxies were observed with ACS/WFC resolution at $z=0.1$.

\section{APPENDIX B: SURFACE PHOTOMETRY OF CONTINUUM MAPS AND DISK/MERGER CLASSIFICATION}

As noted earlier, galaxies in the DYNAMO-HST sample were selected to have rotating, disk-like kinematics based on the classifications given in Green et al. (2014). Recently, Bekiaris et al. (2015) fit kinematic models to many of the galaxies labeled as rotating systems in Green et al. (2014), and confirmed that they are well-described by models of disks. However, in extremely gas rich systems (and many DYNAMO galaxies are likely to be gas-rich, c.f. Fisher et al. (2014)), it is possible for the gas in galaxy-galaxy mergers to quickly form a disk (Robertson et al. 2006), in which case $\mathrm{H} \alpha$ might be a biased kinematical tracer that is decoupled from the motions of the bulk of the stars in a galaxy. Though Bassett et al. (2014) does confirm that gas and stars have similar kinematics, on an galaxy-by-galaxy basis there could be exceptions to this result. To test whether the DYNAMO$H S T$ galaxies are consistent with disk properties, we used data from the FR647M continuum filter to analyze the surface brightness profiles of the starlight in sample, using the code and procedure outlined in Fisher \& Drory (2008). Figure B1 shows the stellar surface brightness profiles of all 10 galaxies in the DYNAMO-HST sample, and (when appropriate) the corresponding best-fit exponential disk models.

HST-DYNAMO Disk Galaxies: Galaxies A04-3, C13-1, D13-5, D15-3, G04-1, G08-5, G14-1 and G20-2 are consistent with having a disk-like exponential surface brightness profile of stars. Of these, galaxies A04-3, C13-1, D13-5, D15-3 and G08-5 have surface photometry that is consistent with a pure exponential disk, while G04-1, G14-1, and G20-2 are consistent with a disk plus a modest $(\mathrm{B} / \mathrm{T}<20 \%)$ bulge. (Note that comparable bulge-to-total ratios are reported for G20-2 \& G04-1 in Obreschkow et al. 2015).

HST-DYNAMO Merging Galaxies: Similar to the other galaxies we attempted to fit traditional exponential profiles G13-1 and H10-2, however the result is an order of magnitude larger residuals from the fits. Galaxies G13-1 and H10-2 have surface photometry that show large, non-central, deviations from single exponential disk or bulge+disk model that is fit to the data. Though low resolution WiFes data suggested rotation (Green et al. 2014), subsequent analysis with higher resolution and deep data from GMOS shows that the kinematic maps of H10-2 are do not have smooth velocity gradients (Bassett et al. 2014), which would suggest they are not rotating disks. G13-1 has kinematics consistent with a rotating disk model (Bekiaris et al. 2015). As we state above, simulations make it clear that when gas fractions are very high, kinematic separation of galaxies into "disks" and "merging systems" can sometimes be misleading (Robertson et al. 2006). Also resolution is also very important, the data used to fit the disk model has $\sim 1-2 \mathrm{kpc}$ resolution (Green et al. 2014), which may not be sufficient in the case of G13-1. In spite of careful pre-selection to isolate rotating disk systems, contamination of DYNAMO-HST by mergers is therefore at the $\sim 20 \%$ level, and G13-1, and H10-2 will be referred to as mergers in the rest of this paper. This is comparable to the merger rate under similar high SFR systems at $z \sim 1-3$ (Förster Schreiber et al. 2009).

This paper has been typeset from a $\mathrm{T}_{\mathrm{E}} \mathrm{X} / \mathrm{LAT}_{\mathrm{E}} \mathrm{X}$ file prepared by the author. 

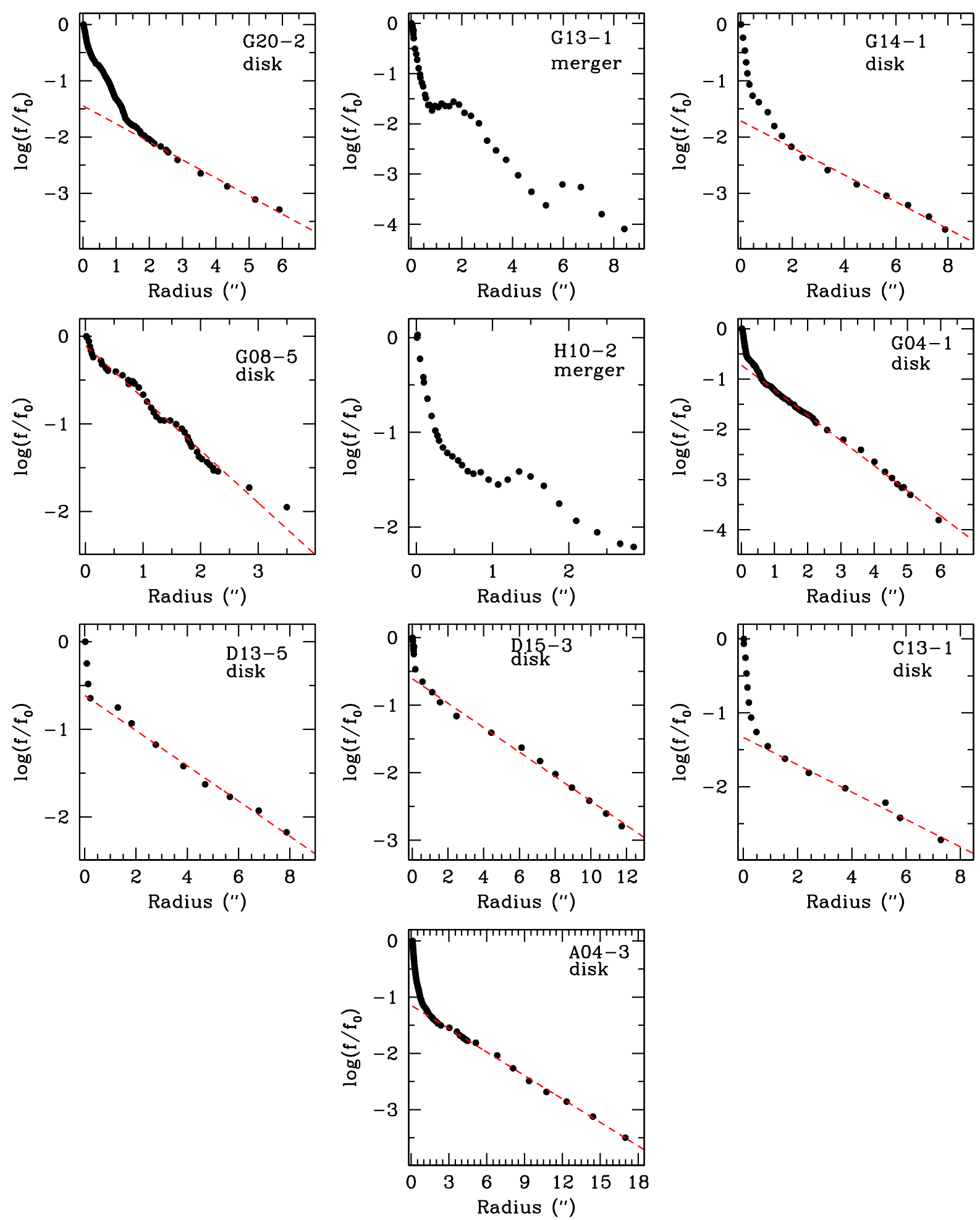

Figure B1. Surface brightness profiles of 6500 Alight for galaxies in the DYNAMO- $H S T$ sample. The galaxies are ordered the same as in Fig. 3. Each surface brightness profile is normalized by the central surface brightness of the galaxy. When appropriate an exponential disk model that has been fit to the data is also shown, represented by a dashed line. The majority of DYNAMO-HST galaxies are consistent both with rotating kinematics and, as shown here, an exponential surface photometry of star light. Therefore multiple lines of evidence suggest that a disk model is appropriate for these galaxies. 